\title{
Local structure-function relationships in human brain networks across the lifespan
}

\author{
Farnaz Zamani Esfahlani ${ }^{1}$, Joshua Faskowitz ${ }^{1,2}$, Jonah Slack ${ }^{1}$, Bratislav Mišić ${ }^{1}$, and Richard F. Betzel ${ }^{1-4}$ 米 \\ ${ }^{1}$ Department of Psychological and Brain Sciences, \\ ${ }^{2}$ Program in Neuroscience, ${ }^{3}$ Cognitive Science Program, \\ ${ }^{4}$ Network Science Institute, Indiana University, Bloomington, IN 47405 \\ ${ }^{5}$ McConnell Brain Imaging Centre, Montréal Neurological Institute, McGill University, Montréal, Quebec, Canada
}

(Dated: July 23, 2021)

\begin{abstract}
The human connectome is the set of physical pathways linking brain regions to one another. Empirical and in silico studies have demonstrated that the structure of this network helps shape patterns of functional coupling between brain regions. To better understand this link between structure and function, a growing number of studies have derived geometric, dynamic, and topological predictors from structural connectivity in order to make predictions about correlation structure. These studies, however, have typically focused on global (whole-brain) predictions using a restricted set of predictors. Here, we investigate a wide range of predictors and shift focus onto predictions of local (regional) patterns of functional coupling. We show that, globally, no individual predictor performs well and, that even the best predictors are largely driven by their ability to predict functional coupling between directly connected regions. We then use the same predictors to make predictions of local coupling and find marked improvement. Notably, the most predictable local FC is linked to sensorimotor regions, which are best predicted by measures based on topological similarity, mean first passage times of random walkers, and the brain's embedding in Euclidean space. We then show that by combining the predictive power of more than one predictor using multi-linear models, we can further improve local predictions. Finally, we investigate how global and local structure-function coupling differences across the human lifespan. We find that, globally, the magnitude of coupling decreases with biological age, which is paralleled by an increase in the number of multi-step pathways. We also show that, locally, structure-function coupling is preserved in higher order cognitive systems, but preferentially decreases with age in sensorimotor systems. Our results illuminate the heterogeneous landscape of structure-function coupling across the cerebral cortex and help clarify its differences with age.
\end{abstract}

\section{INTRODUCTION}

The human connectome constitutes the complete set of neural elements and their anatomical connections to one another 11. At the macroscale, the connectome can be represented as a graph or network whose nodes and edges correspond to brain regions and white-matter fiber tracts [2]. The topological organization of the connectome is non-random and exhibits small-world architecture [3], hubs and rich clubs [2, 4], modules [5], and costeffective spatial embedding [6]. These structural features constrain patterns of inter-areal communication [7-10], inducing correlations in regional activity, i.e. functional connectivity [11, 12.

Estimates of connectomes and functional networks can be obtained easily thanks to easy-to-use processing and reconstruction pipelines [13, 14]. However, linking the two connectional modalities to one another has proven challenging, leading to many different approaches [15, 16. Some of the earliest studies of structure-function coupling constrained generative, biophysical models of brain activity with anatomical connections, noticing that the correlation structure of the synthetic time series output by the model was, itself, correlated with both the

\footnotetext{
* rbetzel @ indiana.edu
}

anatomical network and empirical functional connectivity [7, 8, 17, 18. Other studies have opted for a simpler, albeit amechanistic, approach and computed correlations between anatomical and functional connection weights [2, 19], sometimes using multivariate methods [20].

Although scientifically profitable, both approaches have limitations. Biophysical models, for instance, entail high computational costs that preclude exhaustive searches of parameter spaces. Conversely, statistical and correlative approaches provide limited insight into the mechanisms that support structure-function coupling. Situated between these two extremes, however, are stylized models of interregional communication [12. In general, these models are based on simple dynamical processes, e.g. diffusion [11, epidemic spreading [21, shortest-paths routing [22], whose solutions can be expressed analytically, and entail low computational costs. As a result, this approach allows users to flexibly implement and adjudicate between different models of communication [23].

Broadly, the space of communication models can be organized along a single axis, with models positioned according to how "centralized" or "decentralized" their communication policy is 12, 22. Shortest paths, for instance, are an example of a centralized communication policy in that using a shortest path for signaling requires complete knowledge of a network's global topology. That is, a particle (signal) moving from a source 
region to a target region needs to "know" which nodes are on the shortest path and which connections need to be traversed to stay on that path. In contrast, "decentralized" communication policies require no knowledge and include diffusion processes (random walks) 24] and network navigation [25] - where a particle moves from one node to another according to some greedy policy, e.g. choose the connected neighbor nearest the target in some metric space. Alongside centralized and decentralized models are similarity- 26] and distance-based measures [23], which assess the overlap of brain regions' connectivity patterns or their nearness to one another in space.

Although communication models are becoming increasingly common and have been used recently to study individual differences in phenotypes and traits [27, cognition [28], and for subject identification [29], there remain many open questions and frontiers. First, most studies focus on a select set of communication measures and do not compare the performance of those measures against other models. Second, most studies have focused on using communication models to explain variation in whole-brain functional connectivity. In contrast, several recent studies have shown that structure-function coupling is heterogeneous across the cortex [30, 31, suggesting variation in the underlying communication process and motivating further study. Finally, neither of these questions have been addressed in an applied context. Consider, for instance, the human lifespan. Although many studies have independently documented differences in structural [32 34 and functional connections [35, 36] through development, maturation, and adulthood, how they evolve jointly in terms of communication models and at the level of brain regions is unknown [28, 37.

Here, we address these limitations directly. Using data from the Human Connectome Project 38 and a suite of communication models (predictors) based on both centralized and decentralized processes, topological similarity, and spatial embedding, we show that at the single-subject level, communication models fit at the regional level outperform those fit globally. We also find that predictors based on path length perform poorly, whereas predictors that describe decentralized communication processes perform better. We also show that the variance explained in regional functional connectivity follows a system-specific pattern, with primary sensory systems being more predictable than heteromodal systems. Relatedly, we also find that the distribution of optimal factors vary by brain system. Next, we explore more synergies among predictors, using multi-linear models to predict the weights of functional connections. We find that, among the optimal pairings, a relatively small number of predictors appeared disproportionately often, forming a core set of predictors that, collectively, is essential for predicting regional FC. Finally, we analyze data from the extended Nathan Kline Institute lifespan sample 39. We show that, globally, FC becomes less explainable across the lifespan, irrespective of best pre- dictor. However, we also show that the prevelance of certain predictors vary stereotypically with age and, although FC predictability decreases with age, the regional pattern of predictability was heterogeneous and largely spared systems associated with executive function and introspection (control and default mode networks).

\section{RESULTS}

Here, we explore three interrelated questions: Which communication model best explains observed patterns of FC? How does the optimal model vary across cortex? Does the magnitude of coupling and the optimal model vary over the course of the human lifespan? To test these hypotheses, we analyzed two separate structure-function datasets. The first comes from the Human Connectome Project 38 and includes structural and functional connectivity (SC; FC) data from 100 individuals. The second dataset comes from the Nathan Kline Institute-Rockland lifespan sample [39] and includes SC and FC data from 542 individuals. In the following sections, we analyze cortical networks parcellated into $N=400$ regions of interest 40. For details concerning data processing and network definition, see Materials and Methods.

Our analyses are divided into several sections. In the section Global structure-function coupling is not fully explained by any factor, we investigate individual heterogeneity in terms of which factors best predict whole-brain patterns of FC. Then, in the section Regional structure-function coupling is heterogeneous, we investigate both regional and inter-individual variability in the optimal factor for predicting the $\mathrm{FC}$ profiles of single brain regions. Then, in the section Exploiting synergies among predictors leads to increased explanatory power, we use multi-linear models to explain regional patterns of FC. Finally, in the section Structure-function coupling weakens across the human lifespan, we analyze lifespan differences in structure-function relationships as assessed using communication models.

\section{Global structure-function coupling is not fully explained by any factor}

Recent work has focused on using simple, stylized models to transform sparse SC data into fully-weighted matrices to explain variation in whole-brain patterns of $\mathrm{FC}$ (Fig. 17). In most applications, only a few predictors are investigated, making it difficult to assess the relative performances of different predictors.

Here, we generate a large number of matrices based on SC data from individual subjects. These transformations yield a distinct geometric, topological, or dynamic factor that can then be used to explain variation interregional FC. Broadly, we focused on ten classes of predictors: flow graphs parameterized at different timescales 


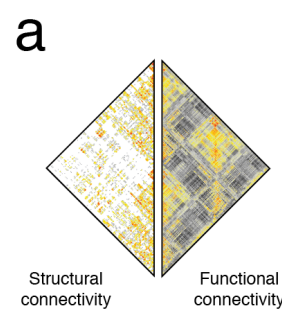

$\mathrm{b}$
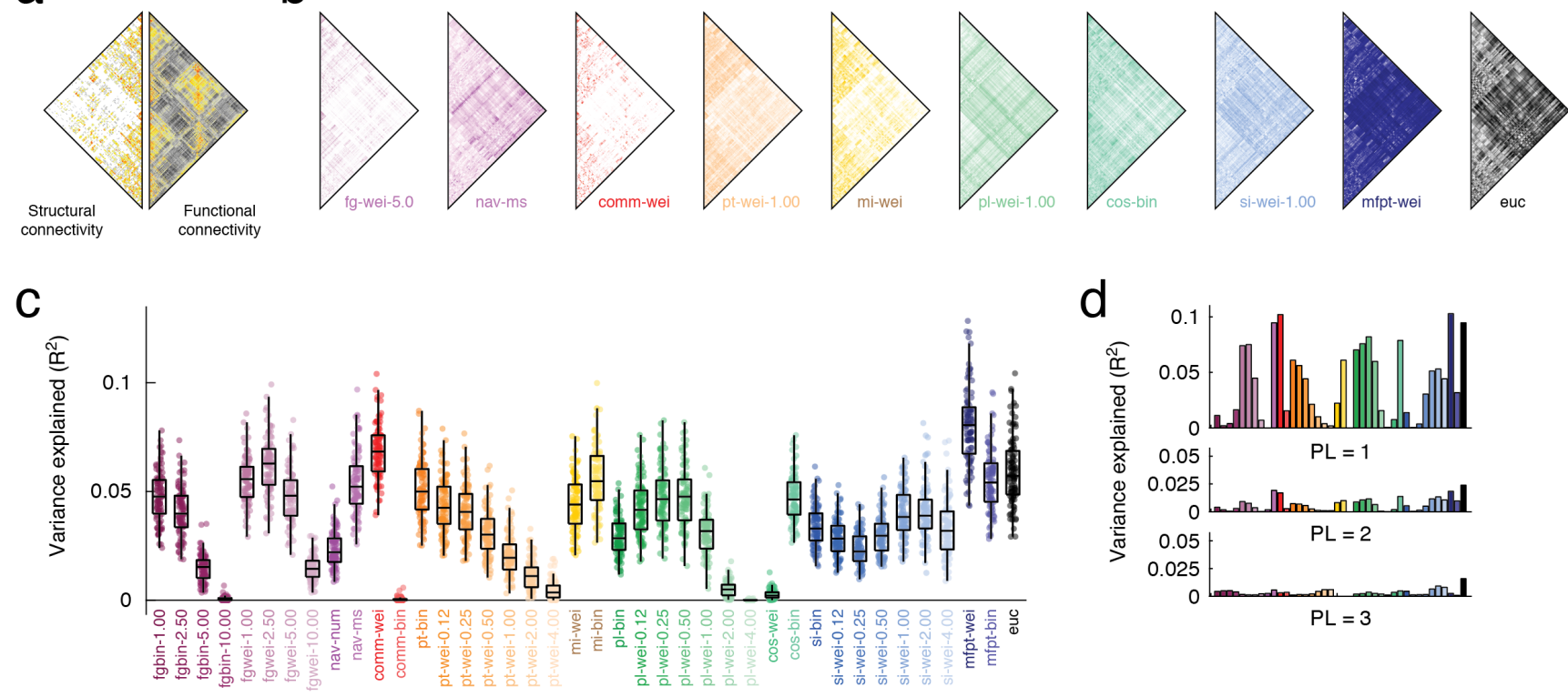

C

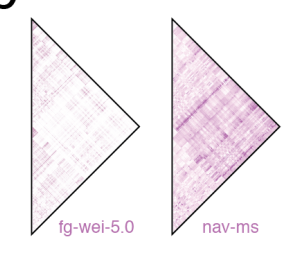

d

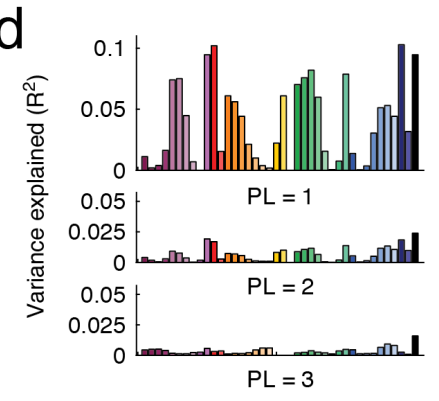

FIG. 1. Whole-brain functional connectivity is heterogeneous and not fully explained by any factor. ( $a$ ) Wholebrain structural and functional connectivity data from the $H C P$ dataset. (b) We used a series of dynamic, topological, and geometric models to transform sparse SC matrices into fully-weighted matrices (predictors). Here, we show examples: flow graphs (fg-wei-5.0), navigation (nav-ms), communicability (comm-wei), path transitivity ( $p t-w e i-1.00)$, matching index ( $m i$ $w e i)$, path length ( $p l$-wei-1.00), cosine similarity (cos-wei), search information (si-wei-1.00), mean first passage time (mfpt-wei), and Euclidean distance $(e u c)$. (c) Variance in whole-brain FC weights explained by factors. Each point represents a subject. (d) Decreases in variance explained as a function of path lengths (hops).

[41, two based on greedy navigation [42, two based on communicability [43, 44, seven based on path transitivity parameterized at different weight-to-cost transformations [11, two based on the matching index [26], seven based on path length parameterized at different weight-to-cost transformations, two based on cosine similarity, seven based on search information parameterized at different weight-to-cost transformations [45, mean first passage times of random walkers [46, and Euclidean distance. In total, we explored 40 different predictors. In Fig. 1 $\mathrm{b}$ we show examples of several predictors for a single subject.

First, we assessed whether the FC variance explained was different from one predictor to another. In general, we found high levels of heterogeneity across predictors in terms of their ability to explain the variance in empirical FC (one-way ANOVA; $F(39)=326.6 ; p<10^{-15}$; Fig. 17). At a single subject level, no predictor explained more than $10.46 \%$ of variance. Aggregating across subjects, the best predictors were weighted mean first passage time (mfpt-wei; $\left.R^{2}=0.079 \pm 0.031\right)$, weighted communicability (comm-wei; $R^{2}=0.068 \pm 0.024$ ), the flow graph estimated at a Markov time of $t=2.5$ (fgwei$2.50 ; R^{2}=0.061 \pm 0.026$ ), and Euclidean distance (euc; $R^{2}=0.058 \pm 0.032$ ). The remaining factors all explained less variance. Note that these general trends persist, irrespective of whether we examine whole-brain connectivity data or connectivity data based on single hemispheres (see Fig. S2). We also tested the statistical significance of the variance explained using a spatially-constrained permutation model [4] (see Fig. S1).

Across all factors, we found that the majority of variance explained can be attributed to one-step (direct) connections (Fig. 1. F). Isolating these connections alone, we found that the average variance explained increased (from $3.5 \%$ to $4.1 \%$; paired sample t-test, $p=8.5 \times 10^{-4}$ ). However, for multi-step paths, the variance explained decreased substantially.

Collectively, these results suggest that whole-brain FC is not well explained by any single factor in isolation $\left(\max \left(R^{2}\right) \approx 0.1\right)$ and that the weights of direct connections are more easily explained than indirect connections. Both of these results are in line with previous studies [11] and motivate further investigation into structurallybased explanatory predictors of FC.

\section{Regional structure-function coupling is heterogeneous}

In the previous section we focused on explaining variance in whole-brain FC using a series of predictors obtained by transforming the SC matrix. In general, we found high levels of heterogeneity and weak correspondence between FC and predictors. In order to achieve 
bioRxiv preprint doi: https://doi.org/10.1101/2021.05.23.445128; this version posted August 22, 2021. The copyright holder for this preprint (which was not certified by peer review) is the author/funder, who has granted bioRxiv a license to display the preprint in perpetuity. It is made available under aCC-BY-NC-ND 4.0 International license.

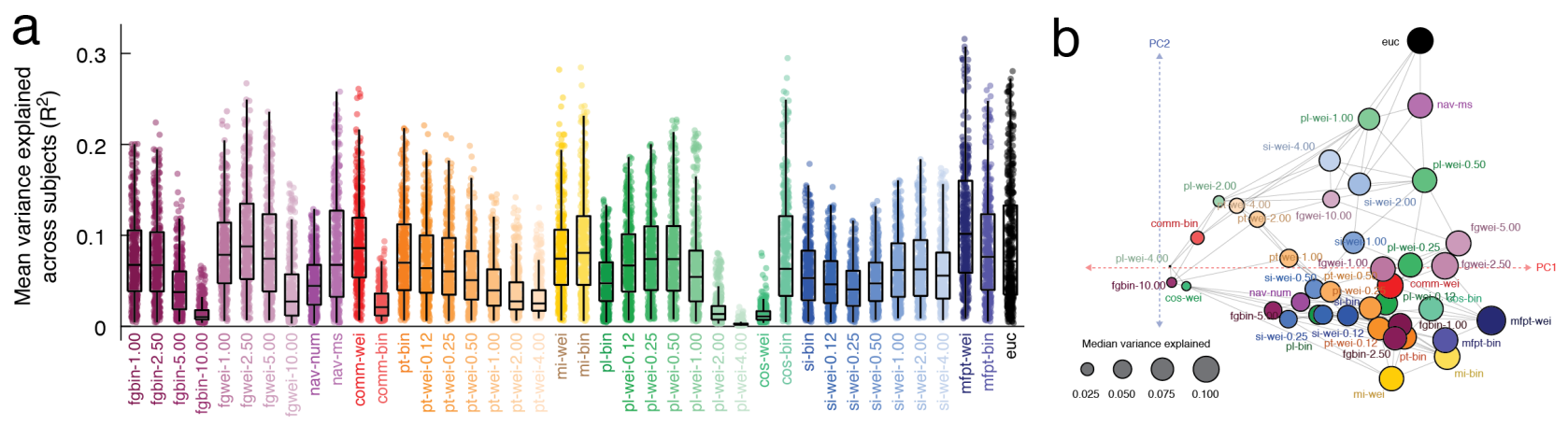

C
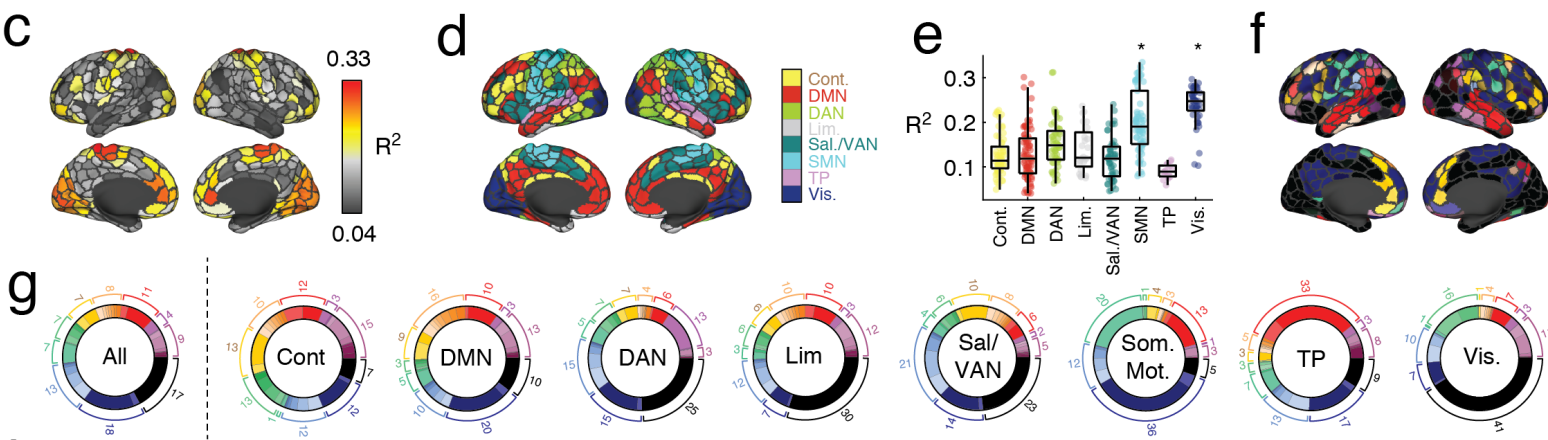

ळ

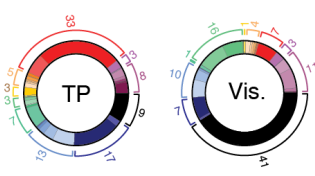

$\mathrm{h}$
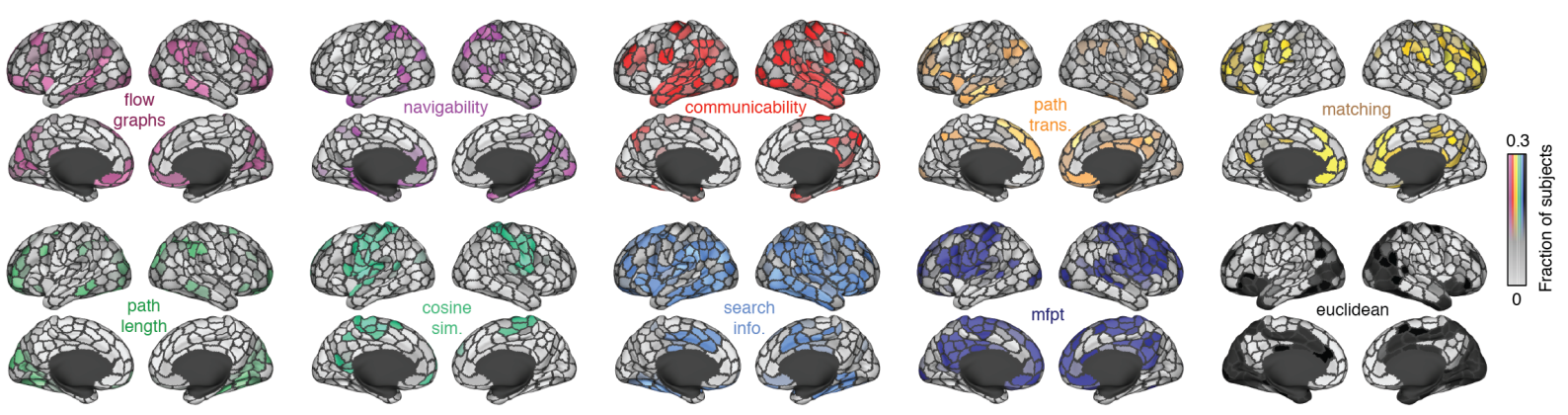

FIG. 2. Explaining regional FC patterns with dynamic, topological, and geometric factors. (a) Distributions of variance explained by different factors. Points represent brain regions and the mean variance explained across the entire cohort. (b) Spatial embedding of predictors. The coordinates were determined using the following procedure: 1) calculating the similarity of regional correlations for every pair of predictors, 2) thresholding this matrix to retain the $k=4$ nearest neighbors for each predictor, and 3) performing a principal component analysis of the thresholded and symmetrized matrix. Coordinates represent the first two principal components, PC1 and PC2. Broadly, predictors that are near/distant to one another in principal component space exhibit similar/dissimilar patterns of regional correlations. In this plot, edges exist between predictors if they are nearest neighbors. The size of points is proportional to the mean variance explained across all brain regions. $(c)$ The maximum variance explained for each brain region by any of the factors. $(d)$ Brain systems mapped onto cortical surface. (e) Maximum variance explained grouped by brain system. $(f)$ Surface projection of the factors that best explain each region's FC pattern. $(g)$ For each subject we calculated the most predictive factor for every brain region. Here, we group these factors at the level of the entire cerebral cortex $(A l l)$ and at the level of individual systems. $(h)$ For each region and factor, we calculated the fraction of subjects for which that factor explained the most variance. Here, we project these values to the cortical surface. Note here that the colors projected onto the surface are continuous; grays correspond to regions where a predictor was optimal for few subjects while brighter colors correspond to regions where a predictor was optimal for many subjects. In all panels the colorscale was capped at a value of 0.3 .

better explanatory power, several recent studies have focused on regional patterns of $\mathrm{FC}$ and explaining variance in $\mathrm{FC}$ from the perspective of individual nodes [30, 31, 48. However, these studies were limited in scope to a select set of predictors. Here, we use the same set of 40 predictors to explain regional patterns of $\mathrm{FC}$ and identify the optimal factors for each region and brain system.

To explain regional patterns of FC, we fit linear models wherein every row (or equivalently column) in the 
FC matrix are explained based on the same row in a predictor matrix. Repeating this procedure for every region, predictor, and subject returns a matrix of $R^{2}$ values whose dimensions are $[400 \times 40 \times 95]$. To visualize these results and for subsequent statistical analyses, we averaged over subjects and plotted the mean variance explained for each region and predictor (Fig. 2a). As in the previous section, we found considerable variability across predictors (one-way ANOVA $R^{2} ; F(39)=141.6$; $p<10^{-15}$ ) but also across regions (one-way ANOVA $R^{2}$; $\left.F(399)=35.4 ; p<10^{-15}\right)$, confirming that both regions and predictors differ from one another in terms of their mean variance explained. We also compared the spatial similarity between predictors (the similarity of the $400 \times 1$ vectors of variance explained at each region) and used an embedding algorithm to assign predictors locations in two-dimensional space based on that similarity (Fig. 2b).

In general, we found that models seeking to explain local (i.e. regional) variation in FC outperform global models. For each region, we calculated the maximum variance explained by any model and found values, in some cases, that exceed $33 \%$ variance (Fig. 2 2 ). Interestingly, the magnitude of variance explained was, itself, variable across cortex and concentrated within specific sets of brain systems (Fig. 2 $\mathrm{d}, \mathrm{e}$ ). In particular, we found that the FC patterns of regions in the somatomotor and visual network were better explained than those of regions in other brain systems (1,000 spin test permutations [49; false-discovery rate fixed at $5 \% ; p_{\text {adjusted }}=0.00368$ ). Irrespective of brain region, we found that Euclidean distance (euc), weighted mean first passage time (mfptwei), weighted communicability (comm-wei), binary cosine similarity (cos-bin), and the length of navigation paths in units of Euclidean distance (nav-ms), were the most common across subjects, being classified as optimal for $16.3,15.8,8.3,6.1$, and 5.1 percent of brain regions. In contrast, the predictors that were least likely to be considered optimal included measures of binary and weighted shortest paths, search information, and flow graphs at long Markov times (See Fig. S3 for complete ranking).

Relatedly, we observed that the predictors associated with the maximum variance explained varied across regions and systems (Fig. 2:). We found that within every brain system certain predictors were overrepresented relative to their baseline rate. Consider the visual system, for instance (Fig. 2 $\mathrm{p}$ ). At the population level, $41 \%$ of visual regions exhibited FC patterns that were best predicted by their Euclidean distance from other regions. In comparison, the FC patterns of only $7 \%$ of control regions were best explained by Euclidean distance (the wholebrain rate is $17 \%$ ). Interestingly, we found that the control and default mode networks diverged from the wholebrain levels at the highest rate, with 16 and 20 of the 40 predictors overrepresented in these systems, respectively (40\% and 50\%). In contrast, sensorimotor systems (somatomotor and visual) overrepresented only seven and five predictors, respectively (17.5\% and $12.5 \%$ ). These observations align with the putative functional roles of these systems - control and default mode are thought to be polyfunctional while sensorimotor systems subtend a narrower set of functions related to processing specific modalities of information.

Indeed, the predictors were differentially associated with brain regions and systems. To better understand exactly which regions were best explained by a given predictor, we grouped predictors into ten broad classes and calculated how each region's FC pattern was best explained by each class of predictor across the 95 subjects (Fig. 22 a). To assess whether these regional patterns of predictor preference were concentrated within distinct brain systems, we averaged their scores by systems [40] and compared these averages with those obtained under a spatially-aware permutation model [47, 49] (1000 permutations; false discovery rate fixed at $5 \%$ leading to adjusted critical value of $\left.p_{a d j}=0.0085\right)$. For instance, we found overrepresented of flow graphs within the control and default mode networks, navigability within the dorsal attention network, communciability in temporoparietal network, path transitivity in default mode, matching index within control network, path length within the visual and control networks, cosine similarity within the somatomotor network, search information within the control network, and mean first passage time within the temporoparietal network. Interestingly, as a predictor Euclidean distance was not overrepresented within any system, suggesting it lacked clear system specificity.

Finally, we calculated for each region the fraction of subjects for whom each of the 40 predictors was optimal. We treated this distribution as a set of features for each region, describing its preference for one predictor or another. Then, we computed the similarity of these feature vectors for every pair of brain regions, resulting in $400 \times 400$ correlation matrix, which we then clustered using modularity maximization. We found evidence of ten consensus communities, four of which were large and were investigated further (Fig. S4). In general, each of these four communities disproportionately represented a single specific predictor. Namely, weighted mean first passage time (cluster 1), Euclidean distance (cluster 2), weighted communicability (cluster 3 ), and weighted matching index (cluster 4) (Fig. S4.).

We also repeated this process using structural connection weights instead of derived predictors (see Fig. S5. We found that, while the two techniques generated similar regional patterns of explained variance (mean similarity of $r=0.36 \pm 0.08$ ), the predictors generally outperformed structural connectivity, with the FC of $67.5 \pm 0.06 \%$ of regions being better explained by predictors derived from the SC matrices than the SC matrix itself.

Collectively, these results suggest that global models of interregional communication may fail to account for regional preferences in communication patterns. By fitting explanatory models at the level of regions, we can expose 

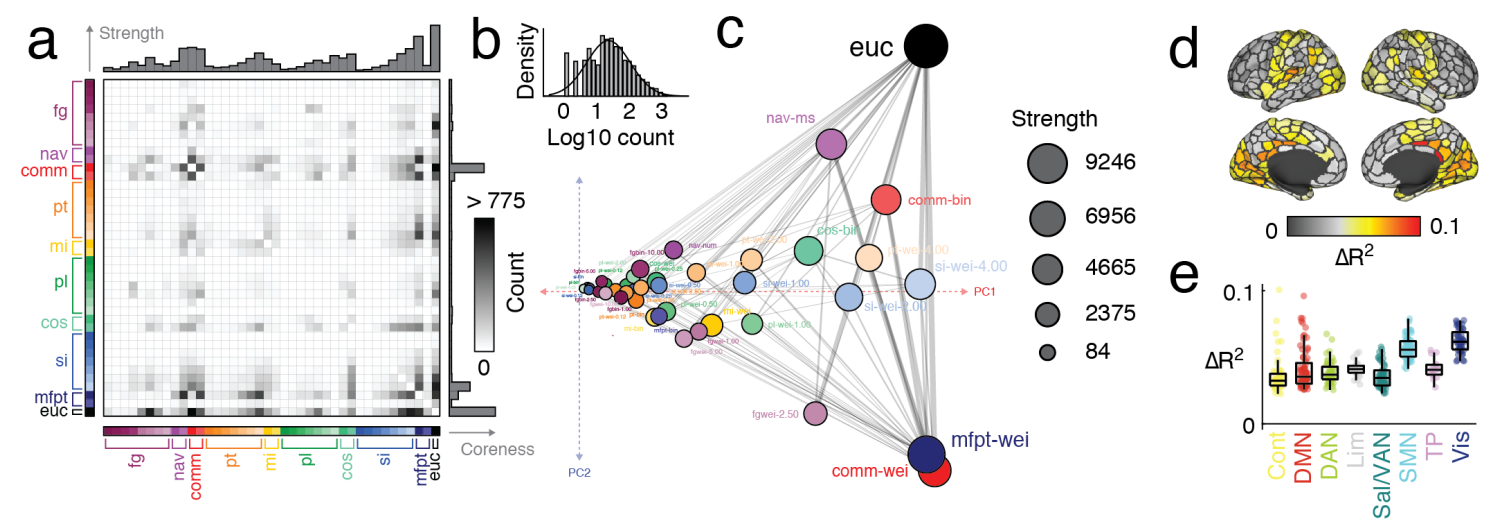

FIG. 3. Pairwise synergies between predictors. We used two-predictor multi-linear models to predict regional patterns of FC and identified the optimal pair of predictors for each region and each subject. We then counted how frequently each pair of predictors appeared in the set of optimal predictors. This procedure yields a symmetric matrix of counts $(a)$. The counts were approximately log-normally distributed $(b)$. The matrix could be modeled as a graph and each node's (predictor's) coreness could be directly calculated (node positions based on principal components analysis of count matrix) (c). In this plot, coordinates were determined by: 1) thresholding the count matrix to retain, for each predictor, its $k=4$ nearest neighbors, and 2) performing a principal component analysis on the thresholded and symmetrized matrix. Here, the coordinates represent the first two principal components, PC1 and PC2. Predictors that are near/distant from one another in principal components space pair with similar/dissimilar sets of predictors when improving $R^{2}$. Predictors are joined by an edge if they are considered nearest neighbors. We also calculated the mean regional improvement in $R^{2}$ from using the multi-linear model versus the model with a single predictor. $(d)$ Improvement $\left(\Delta R^{2}\right)$ projected onto the cortical surface. (e) Improvement grouped by canonical brain systems.

these preferences and heterogeneity across the cerebral cortex in terms of regional predictability.

\section{Exploiting synergies among predictors leads to increased explanatory power}

In the previous two sections, we demonstrated that at the whole-brain level, measures of communication explained a small fraction of variance in FC, but could be improved upon by modeling FC at the level of individual brain regions. In both cases, we modeled FC linearly in terms of one predictor and a constant. However, several studies have examined the extent to which combinations of predictors, e.g. search information and Euclidean distance [11, 23, 31] led to an improvement in variance explained when combined in a multilinear model. Here, we focus on local models while simultaneously building upon those earlier studies to systematically explore all possible combinations of terms.

We constructed linear models of FC based on pairs of predictors. With 40 predictors this yields $40 \times 39 / 2=780$ unique dyads, which we used to explain regional $\mathrm{FC}$ variance. To reduce the total number of dyads, we performed the following greedy analysis. For each subject and region, we identified the predictor that explained the greatest fraction of variance in its FC. Then, of the remaining 39 predictors, we identified the one that, when included in the multilinear model, yielded the greatest performance improvement. Then, we counted how fre- quently specific pairs of predictors appeared together in the multilinear models and summarized the results as a square predictor $\times$ predictor matrix (Fig. 3 3 ). Interestingly, we found that the counts followed a heavy tailed distribution, such that a small fraction of predictor pairs appeared disproportionately more frequently than others (Fig. 3b). When we considered the marginal distribution (Fig. 3 a, bar plot at the top of the matrix), we found that Euclidean distance, weighted mean first passage time, weighted communicability, weighted search information (with $\gamma=4$ ), and navigability participated in the most dyads, suggesting that these factors, when paired with others, are important for explaining regional patterns of FC.

To better understand the interrelationships among predictors, we modeled the matrix in Fig. $3 \mathrm{~h}$ as a graph, where nodes and edges correspond to predictors and the frequency with which predictor pairs appear, respectively (see Fig. 3c for an embedding of the network in twodimensional space). Upon visual examination of the connectivity matrix, it appeared that a small fraction of predictors broadly interacted with others while the remaining predictors weakly interacted with one another. This type of organization is hallmark of core-periphery mesoscale structure, where a densely-connected core of nodes projects to a sparsely connected periphery. In this context, the "core" refers to pairs of predictors (metrics) that frequently appear together in two-term multilinear models. The same core predictors may sometimes be paired with peripheral predictors, but peripheral predictors are 
infrequently paired together. That is, the core is comprised of predictors that exhibit strong synergies in their ability to predict FC patterns; the periphery is comprised of predictors that exhibit relatively weak synergies. To test whether this type of structure was present, we applied a core-periphery detection algorithm that, rather than a binary classification of a node as "core" or "peripheral", assign each node a parameterized and continuous measure of coreness. Here, we systematically varied the two parameters $-\alpha$ and $\beta$ - which control, roughly, the smoothness of the distinction between core and periphery and the number of nodes in the core, respectively. We aggregated coreness scores over the top $5 \%$ of parameter pairs to obtain a mean coreness score for every node (see Fig. S6). As expected, the predictors with the highest levels of coreness included Euclidean distance, weighted communicability, weighted mean first passage time, and search information (with $\gamma=4$ ) (Fig. 3 p; right margin). With the exception of Euclidean distance, all of these measures are based on diffusive, decentralized dynamics. Mean first passage time and search information concern random walks over a network, while communicability is associated with the ensemble of multi-step walks through a network. In contrast, measures based on shortest-paths routing (weighted and binary shortest paths) fall squarely in the periphery.

In the previous analyses, we examined synergies between pairs of predictors. Specifically, we focused on how synergies vary across the brain, which regions are associated the greatest improvements, and what pairs of predictors drive these improvements? First, we compared the increase in explained variance $\left(\Delta R^{2}\right)$ as a result of including the second predictor. As expected, all changes in variance explained were positive (Fig. 3d) and were largely concentrated in somatosensory systems (Fig. 3e), suggesting that the biggest increases were associated with regions and systems whose baseline $R^{2}$ was among the greatest prior to introducing a second predictor. In the supplementary material we repeat this analysis using the adjusted $R^{2}$ to confirm that changes in variance explained are not simply a consequence of the additional parameter in the linear regression model (Fig. S7).

For completeness, we all considered the effect of combining all predictors into the same linear model. Note that many of the predictors are correlated, making it difficult to assess their unique contributions. However, we note that including all predictors yields $R^{2}$ values that are, on average, $2.4 \pm 0.6$ times greater than the best pairs of predictors. The regions that benefit the most from the inclusion of all predictors fall within default mode and salience/ventral attention systems, and, in some cases, exhibit increases in $R^{2}$ that are ten times that of the best predictor pair. The results of this additional analysis are summarized in Fig. S8.

In parallel, we repeated the above analysis using principal components derived from predictors rather than the predictors themselves. This procedure helps address concerns related to correlated predictors. In general, the re- sults of this analysis converge with those reported here. See Fig. S9 for a summary of these results.

Collectively, these results demonstrate that improvements gained by using multiple predictors to explain FC have distinct spatial topography, favoring unimodal sensory systems. Moreover, even with multiple predictors, the predictability of FC in heteromodal cortices improves little.

\section{Structure-function coupling weakens across the human lifespan}

In the previous sections we systematically evaluated the utility of different structural predictors for explaining variance in regional patterns of FC. Those analyses were carried out using data from the Human Connectome Project and included subjects of, roughly, the same age range (young adult; 18-30 years). In this section, we use data from the enhanced Nathan Kline Institute-Rockland sample, which comprises 542 individuals from the Rockland, NY community whose ages range from childhood through senescence (7-85 years). Specifically, we focus on the magnitude of structure-function coupling across the lifespan and differences in the optimal predictor as a function of age.

First, we assessed how differences in global structurefunction correspondence varied with age. To do this, we calculated the maximum $R^{2}$ for each participant across all predictors. Then, to rule out the possibility that interindividual differences in variance explained is related to differences in sex, time of visit (for data acquisition), or global network properties like total weight and binary density, we regressed these values out of each subjects' $R^{2}$ value. The residuals obtained following this procedure are, by definition, orthogonal to those nuisance variables. Finally, we calculated the linear correlation of these residuals with subjects' ages and observed that the two were significantly associated with one another $\left(r=-0.27 ; p=2.5 \times 10^{-10}\right) ;$ Fig. 4 a , suggesting that the magnitude of structure-function correspondence decreases monotonically with age. Globally, the most common optimal predictors of FC were Euclidean distance (51\% of participants) and weighted mean first passage time (37\%) (Fig. 4b).

The previous analysis focused on global coupling between structure and function. Next, we investigated agerelated differences in structure-function coupling at a local (regional) level. As with the global analysis, we regressed out the effect of sex, time of visit, and global network properties. Because we were examining effects at the level of individual nodes, we also regressed out the effect of nodes' binary and weighted degrees. First, we asked whether the prevalence of certain predictors varied with age. For each region, we identified the predictor that best explained its regional pattern of $\mathrm{FC}$ and, for each subject, calculated the fraction of regions best explained by each factor. We then grouped subjects into percentile- 

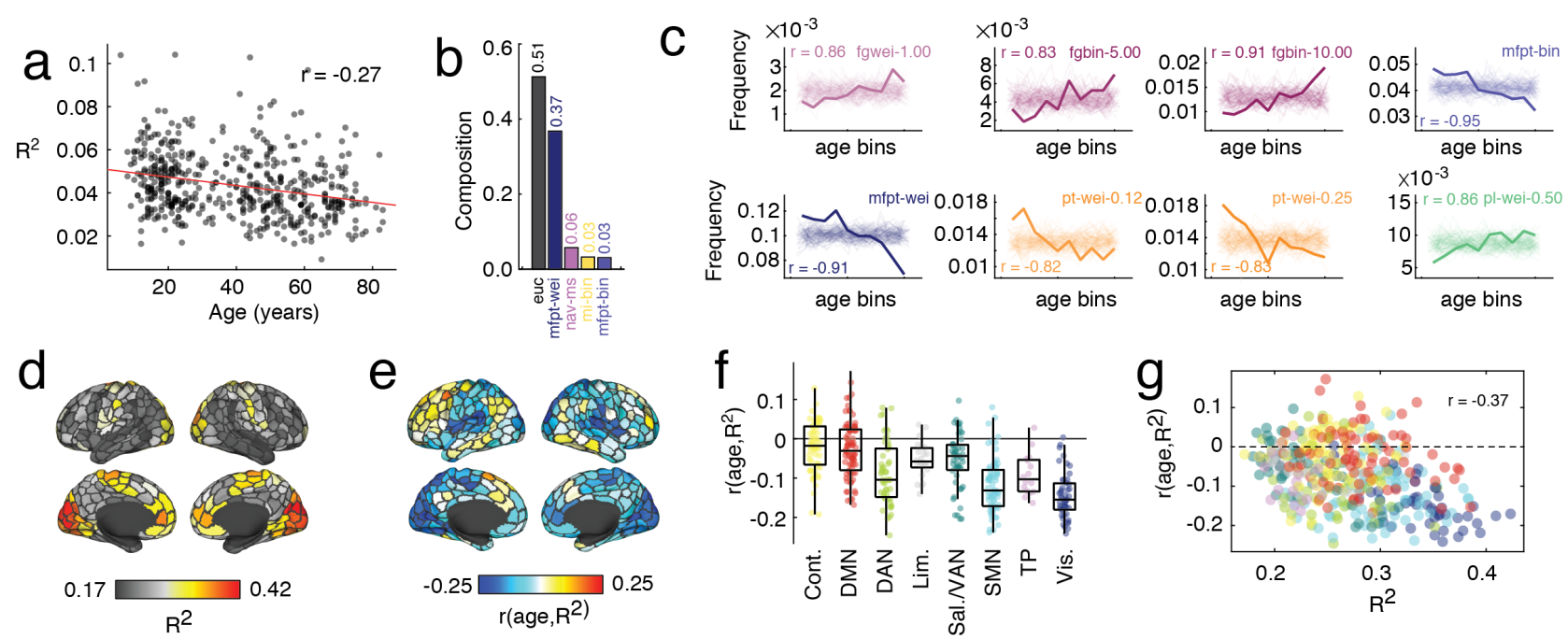

FIG. 4. Lifespan variation in structure-function coupling based on communication models. (a) Decreases in wholebrain variance explained across the lifespan. (b) Distribution of optimal predictors for each subject across the lifespan. (c) We grouped subjects into 10 bins based on their ages and calculated, for each bin, the frequency with which different predictors were optimal for explaining regional patterns of FC. $(d)$ Whole-brain pattern of variance explained. $(e)$ Correlation of variance explained with age projected onto the cortical surface. $(f)$ Correlation of variance explained grouped by brain system. ( $g$ ) Scatterplot of variance explained versus the correlation of variance explained and age.

based age bins (10 bins in the main text; see Fig. S10 for reproducibility of results with different numbers of bins), and found that eight predictors from four different families varied significantly across the lifespan. These included binary and weighted flow graphs, binary and weighted mean first passage time, weighted path transitivity, and weighted path length (Fig. 44). Notably, when we calculated the maximum variance explained at each region, we found a pattern that was highly correlated with that of the HCP dataset $(r=0.73$; Fig. $4 \mathrm{~d})$.

Next, we asked whether the maximum variance explained by any predictor - a measure of structurefunction coupling - varied with age. We found a hemispherically symmetric (Fig. 44) correlation pattern in which most regions, mirroring the global pattern, decreased with age. Interestingly, the spatial pattern of correlations was system-specific, with negative correlations significantly concentrated within somatomotor and visual systems (Fig. 4f). Interestingly, the relationship between variance explained and its correlation with age was negative, so that regions with high levels of structure-function coupling in normative adults were more likely to decrease with age (Fig. $4 \mathrm{~s}$ ). Note that we also found systemspecific correlations of $R^{2}$ with measures of intelligence, although these values were overall much weaker in magnitude (see Fig. S11). We include thresholded surface maps of the correlations between $R^{2}$ with both age and intelligence in the supplementary material S12). Additionally, we assess the effect of data quality on reported results and find that they hold after excluding subjects on the basis on in-scanner motion and temporal signal to noise (Fig. S13).
Collectively, these results suggest that the interrelationship of structural and functional connectivity covaries weakens with age. Notably, the areas that exhibit the greatest reductions include sensorimotor systems, which are among those with the strongest coupling to begin with. Heteromodal systems, like default mode and control networks, on the other hand, exhibit subtle reductions in coupling magnitude and, in some cases, even increase with age. Our findings point to heterogeneous differences in the complex relationship between the brain's physical wiring and its intrinsic functional organization.

\section{DISCUSSION}

Here, we aimed to address two questions about structure-function coupling in brain networks. What structurally-derived measure does the best job predicting FC? Second, how does the best measure vary locally, from one region to another? As a final application, we considered how the optimal predictor varies with biological age in a large lifespan dataset. We found that predictions of whole-brain FC were poor, irrespective of predictor, and could be improved upon by making predictions at a local, i.e. regional level. At this scale, the optimal predictor varied by region, with Euclidean distance and weighted mean first passage time among the best. Consistent with previous reports, the spatial patterning of structure-function coupling favored sensorimotor cortices. We then explored synergies between predictors and their inclusion in multi-linear models. Again, we found 
that Euclidean distance and weighted mean first passage time, along with measures of communicability and, in some instances, search information and path transitivity, exhibited strong synergistic relationships such that their joint inclusion in a model tended to support improvements in functional variance explained. Finally, we examined structure-function coupling across the human lifespan. We found that, globally, the magnitude of coupling decreased with age, an observation driven by decreases in local coupling of visual and somatomotor systems. Structure-function coupling in higher-order cognitive systems, including the control and default mode networks, went largely unchanged with age and, in some cases, even increased.

\section{Communication processes and sparse-to-fully weighted transformations of SC}

Many studies have attempted to link patterns of structural and functional connectivity to one another. At one extreme are studies that investigated neurobiologically realistic models, e.g. neural mass models (NMMs), whose parameters correspond to biophysical constants and generate time-varying voltage traces from neuronal populations [7, 17, 24, 50, 51. These models offer a mechanistic description of how structural links constrain brain dynamics and give rise to cohesive and correlated activity. The performance of neural mass models can be improved upon by allowing for regional heterogeneity in parameters, matching additional features of empirical brain activity, including separation of dynamic timescales and the patterning of time-varying connectivity [52, 53]. However, NMMs come at a high computational cost, limiting the possibility of performing exhaustive parameter searches or fitting the complete set of parameters at a subject-level.

At the other extreme are studies that link structure to function by directly comparing the weights of connections. In one of the earliest studies of the human connectome, the authors showed that structural weights (estimated as a length and volume normalized streamline count) and their corresponding functional connections are correlated, both globally and for select seed regions [2. Similarly, 54] demonstrated that coherent patterns of spontaneous and task-evoked activity in the macaque oculomotor system are supported by anatomical connections. These types of correlative relationships are found at other spatial scales using invasive reconstruction and imaging techniques applied to mode organisms. For instance, in [55], the authors used "barcoding" to reconstruct cellular-level synaptic connectivity and linked connection weights to the correlation structure of spontaneous activity recorded using widefield fluorescence imaging. Broadly, these approaches demonstrate that, for structurally connected neural elements, their anatomical connection weight is correlated with the similarity of their recorded activity. This approach for studying structure-function relationships, however, is limited in that comparisons between connection weights can only be carried out for directly connected pairs of neural elements. If two cells, populations, or regions are not directly connected, then it becomes impossible to make a prediction about its functional coupling.

The results reported here are situated between these two extremes and adopt useful principals from each while avoiding some of the pitfalls [12. Specifically, we focus on predicting the weights of functional connections by transforming the sparse SC matrix into a fully-weighted (and possibly signed) matrix. Some of these transformations incorporate elements of dynamics. Flow graphs, for example, embed the probabilistic flow of random walkers between two nodes into the edges of a graph [41. Other transformations embody communication policies that the brain could conceivably implement as means of transmitting a signal/information from one region to another. Shortest paths are an example of a centralized communication process, in that to take advantage of these paths would require global knowledge (a centralized pool of information) of the network's shortest path structure. In contrast, decentralized processes like diffusion/random walks or navigation evolve without the need for any additional information [11, 45. Path transitivity and search information blend these two concepts, treating shortest paths as fixed constructs, but asking how easily they could be traversed passively by a knowledgeless random walker. Other transformations represent geometric relationships between nodes or the topological similarity of their structural connectivity patterns.

Practically, using matrix-based predictors to explain FC has many advantages. Unlike biophysical models, all of the predictors studied here can be generated in seconds of computation time, reducing the computational burden associated with realistic models. However, this improvement in computational complexity does not require that we jettison all information about dynamics; as noted earlier, many of the matrices provide summary information about dynamical processes. This approach also circumvents the issue of missing connections. Unlike correlative methods that can only compute the similarity of existing structural and functional connections, this approach transforms the sparse structural matrix into a fully-weighted matrix, where every entry can be, in principle, non-zero. This allows for a more direct comparison. Lastly, previous studies of identical datasets have shown that the matrix predictors tend to outperform even biophysical models in terms of matching empirical patterns of FC [8, 11].

Here, we extend the matrix-based prediction approach from a global, whole-brain level to a the level of individual brain regions. Our approach builds upon other recent studies that examined heterogeneity in coupling patterns across regions [30, 31] and systems [48. Despite differences in dataset, parcellation, and choice of predictor, our results are closely aligned with those of previous studies, which reported strong coupling in sensorimotor cortices 
and weaker coupling in heteromodal regions. Interestingly, heteromodal cortex includes regions that expanded dramatically over the course of mammalian evolution [56] and others that develop at a protracted rate [57, leading to the hypothesis that their interregional connectivity may be shaped by different organizational principles than regions in unimodal cortex and serving as a possible explanation for the weakened structure-function coupling.

Of course, there are serious tradeoffs associated with modeling $\mathrm{FC}$ in terms of matrix-based predictors derived from SC. Namely, it sacrifices the neurobiological plausibility of NMMs for computational ease and the interpretability of direct structure/function correlations in order to generate fully-weighted matrices. Additionally, matrix-based predictors fundamentally lack a temporal dimension. That is, they compress information about dynamical processes, e.g. diffusion, navigation, shortest paths routing, into matrix form, the precise temporal evolution of those processes is lost. In contrast, biophysical models document the temporal evolution of activity, generating spike trains, voltage traces, or hemodynamic signals from cells, populations, or regions $[5860$. These temporal data provide an additional target for modeling studies; rather than simply matching the correlation structure of brain activity, can a model also replicate its time-varying features? Indeed, while recent work has begun to investigate time-varying structure-function coupling [53, 61,63], future studies are necessary.

In summary, there exists a spectrum of methods for assessing and modeling structure-function coupling in empirical data [18, 64-70]. Each method possesses a distinct set of advantages and disadvantages, such that some research questions are better addressed by one method and not another. For instance, biophysical models offer a neurobiological account of the relationships between structure and function while making populationlevel time courses available for analysis. On the other hand, the complexity of biophysical models might make them unappealing for studies where the aim is to generate a biomarker of structure-function coupling. In that case, a simple correlation between $\mathrm{SC}$ and $\mathrm{FC}$ weights may suffice. In general, these methods should be viewed as complementary rather than adversarial, affording researchers the opportunity to examine data under different sets of assumptions, across spatial scales, with varying levels of neurobiological realism, and using different computational tools.

\section{Shortest paths or diffusion; centralized or decentralized processes?}

Path-based metrics are often used as markers to compare populations of individuals in an effort to distinguish clinical subjects from controls [71] or to be linked with a continuous measure, e.g. intelligence scores [72. These metrics include the well-known characteristic path length - the mean number of steps in shortest paths over all pairs of nodes - and efficiency, the mean reciprocal of the shortest path lengths [73].

Although these measures are commonplace in network neuroscience, they implicitly prioritize shortest paths as the communication routes between brain regions. Although superficially this seems like a reasonable assumption, other studies have cast doubt on these findings, noting that the backbone of shortest paths involves only small fraction of network edges [21, 74 and that, for a brain to use shortest paths for communication, requires that it has global knowledge of its shortest path structure [11, 22], which is not obviously biologically plausible.

Recently, however, a growing number of studies have presented alternative and decentralized communication models. These include models of diffusion and random walk dynamics [24, 75, epidemic spread [21, 76], more complicated models that allow for an interpolation between centralized and decentralized processes [22, and navigation models [42, 77]. Unlike shortest paths, these communication processes evolve using local knowledge - the next step depends on the weights of edges (random walks) or the distance of directly connected neighbor from the eventual target.

Here, and in agreement with other studies [11, we find that shortest paths structure (both binary and weighted) do a poor job in recapitulating patterns of FC. In contrast, measures like mean first passage time, communicability (which emphasizes not just the single shortest path in a network, but all walks of all lengths), and others derived from navigation appear near the top of the list in terms of frequency. Although these observations do not conclusively demonstrate that shortest path structure plays no role in communication processes, they do support the hypothesis that decentralized measures may play an outsized (and possibly underappreciated) role in shaping interregional communication processes. We note, however, that under certain circumstances, e.g. biased random walks or navigation strategies [42], may access a network's shortest paths.

\section{The impact of space on structure-function coupling}

One of the long-standing observations about brain networks is that their architecture is shaped, in part, by the space in which they are embedded [78. The tight statistical relationship between distance and presence/absence of structural connections and their weights holds at virtually all scales [74, from cellular-level connectomes [79, to areal maps [80], to non-invasive imaging with MRI 81. This relationship also holds for functional imaging data [82, although the coupling between space and FC is statistically less severe [83, 84].

The observation that SC and FC are both constrained by space has lead to speculation that at least some fraction of variance in structure-function coupling can be attributed to their joint embedding in Euclidean space. Indeed, previous studies have shown that structure- 
function correlations are attenuated after regressing out the effects of Euclidean distance [11, 23], though the resulting correlations suggest that there remains some residual relationship.

Here, we adopted a local perspective on structurefunction relationships and directly compared different predictors with one another. This allows us to test the extent to which Euclidean distance outperformed any of the other network measures for predicting FC. Indeed, we found that Euclidean distance was always among the best measures. This was true for predicting FC globally in both the HCP and NKI datasets, as well as the analysis of local connectivity. However, our local analysis revealed that, for many regions, especially those in control, somatomotor, and temporoparietal networks, Euclidean distance was outperformed by other measures. On the other hand, Euclidean distance was overrepresented as an optimal predictor within the visual, salience/ventral attention, and dorsal attention networks.

These observations suggest that the impact of spatial relationships on $\mathrm{SC}$ an $\mathrm{FC}$ and their statistical coupling to one another is heterogeneous and, to some extent, system-specific.

\section{Differences in structure-function coupling with age}

The progression through development, maturation, and senescence is one of the most profound and shared human experiences. It is accompanied by increased diffusivity of task-evoked brain activity 85] and reductions in system segregation 35, 36. Similar differences occur structurally, with reductions in modularity 86] and increased characteristic path length 32 . Less is known about how structural and function differences occur in parallel, and especially at the local scale [30.

Here, we investigate lifespan differences in structurefunction coupling, and find that with age, the global correlation is reduced. At the local scale, these differences are paralleled by reductions in several predictors, notably weighted mean first passage time (the other predictors that exhibit lifespan differences do so at a much slower rate). Mean first passage time refers to the number of steps in a random walk when a random walker starting at node $i$ is likely to have visited node $j$. Importantly, mean first passage time is a descriptor for a diffusive (random walk) process. The same is true for flow graphs and path transitivity, which vary systematically with age, albeit weakly. Based on these findings and along with the observation that the magnitude of global structure-function coupling decreases with age, we speculate that, decentralized patterns of interregional communication may degrade over the human lifespan, prompting a decoupling of functional connectivity from structure.

However, there are several potential limitations of this analysis. Specifically, there exist alternative interpretations of our results that are difficult to rule out conclusively. Here, we interpret $R^{2}$ as a measure of how strongly or weakly structural and functional connectivity are coupled to one another and that age-related differences in $R^{2}$ reflect shifts in network-level communication patterns or policies. Aging, however, is accompanied by systematic differences in other brain and physiological measurements [87, including level of in-scanner motion [35, 88], respiratory and cardiac signatures, and neurovascular coupling [89]. Here, we adopted a conservative approach to addressing motion-related issues, excluding from analysis any subjects whose in-scanner motion exceeded a threshold. This approach, however, may inadvertently exclude subjects with usable data. Addressing issues related the neurovascular coupling, on the other hand, presents a greater challenge, as how best to assess and correct for subject- and region-level coupling patterns remains an open frontier in aging neuroscience [90, 92]. This topic should be the focus of future studies.

\section{Future directions and limitations}

This study suffers from a number of limitations. Streamline tractography provides an estimation of the underlying white matter that is potentially hampered by biases involving complex fiber geometry and limitations given the inverse problem that the methodology aims to solve [93. For instance, our current study focuses on cortico-cortical pathways, excluding subcortical nuclei and the cerebellum from analysis. While the justification for this exclusion is straightforward - limitations of whole-brain diffusion imaging and tractography make it challenging to accurately resolve the connections of small structures in the basal ganglia [94, 95] it nonetheless leaves open the possibility that our results will change with the inclusion of these structures. Future studies should examine whole-brain communication processes and include structures from the subcortex and cerebellum 96.

Another potential limitation concerns the breadth of matrix-wide predictors. Here, we examine 40 predictors, each of which of represent a dynamical, topological, or geometric factor that could explain patterns of FC. However, there are other predictors that could, in principle, be explored here but due to the scope of the paper are not. In addition, there are other methods, including spectral decompositions [69], deep learning [67, and embedding models [70, 97, that can lead to high levels of structure-function coupling, but present little mechanistic insight. Future studies should investigate alternative predictors and other methods in greater detail, seeking to merge machine-learning and neurobiological accounts of structure-function coupling.

An additional concern is related to age differences in structure-function coupling and the possibility of a floor effect. Specifically, we found that regions with low baseline levels of $R^{2}$, our measure of structure-function coupling, were among those that decreased the least with age. While we cannot definitively rule out the possibil- 
ity of a floor effect, we note that regions with the lowest structure-function coupling magnitude have values near $R^{2}=0.17$, suggesting that, at least in principle, coupling could decrease further.

Interestingly, while the regional patterns of structurefunction coupling in NKI and HCP datasets were correlated, the magnitude of coupling was noticeably stronger. While the origins of this difference remain unclear, possible explanations include differences in sample size $(N=$ 95 in $\mathrm{HCP}$ versus $N=542$ in $\mathrm{NKI}$ ), heterogeneity, and community representativeness. Other possibilities include differences in data quality and preprocessing strategies. In the NKI dataset, functional and structural networks were estimated using a volumetric parcellation in subject anatomical space. In contrast, the HCP data was processed using surface-based analyses. Indeed, recent studies have identified notable differences between these two image processing pipelines [98. Future studies should investigate this possibility in greater detail, documenting the effect of different processing pipelines on local structure-function coupling.

A final limitation concerns the possibility that the reported correlations between $R^{2}$ and age could be attributed to a floor effect. That is, any regions that start with low $R^{2}$ early in life necessarily have less room to decrease over the course of the lifespan, whereas regions that start with high levels of $R^{2}$ have more room to decrease. Although we show that the fraction of regions that may be susceptible to this effect is small, the spatial pattern is correlated with the reported $R^{2}$ vs age correlation map (see Fig. S14). Disentangling true agerelated differences from floor effects is challenging and may require the use of an unbounded measure other than $R^{2}$ for assessing structure-function correspondence. Future work should investigate these potential confounds in greater detail.

\section{MATERIALS AND METHODS}

\section{Datasets}

\section{Human Connectome Project}

The Human Connectome Project (HCP) dataset 38 consisted of structural magnetic resonance imaging (T1w), resting state functional magnetic resonance imaging (fMRI) data, as well as diffusion magnetic resonance imaging data (dMRI) from 100 unrelated adult subjects. These subjects were selected as they comprised the "100 Unrelated Subjects" released by the Human Connectome Project. After excluding subjects based on data completeness and quality control (see Quality Control), the final subset utilized included 95 subjects ( $56 \%$ female, mean age $=29.29 \pm 3.66$, age range $=22-36)$. The study was approved by the Washington University Institutional Review Board and informed consent was obtained from all subjects. A comprehensive description of the imag- ing parameters and image preprocessing can be found in 99. Images were collected on a $3 \mathrm{~T}$ Siemens Connectome Skyra with a 32-channel head coil. Subjects underwent two T1-weighted structural scans, which were averaged for each subject $(\mathrm{TR}=2400 \mathrm{~ms}, \mathrm{TE}=2.14 \mathrm{~ms}$, flip angle $=8^{\circ}, 0.7 \mathrm{~mm}$ isotropic voxel resolution). Subjects underwent four resting state fMRI scans over a two-day span. The fMRI data was acquired with a gradient-echo planar imaging sequence $(\mathrm{TR}=720 \mathrm{~ms}, \mathrm{TE}=33.1 \mathrm{~ms}$, flip angle $=52^{\circ}, 2 \mathrm{~mm}$ isotropic voxel resolution, multiband factor $=8$ ). Each resting state run duration was 14:33 min, with eyes open and instructions to fixate on a cross. Finally, subjects underwent two diffusion MRI scans, which were acquired with a spin-echo planar imaging sequence $(\mathrm{TR}=5520 \mathrm{~ms}, \mathrm{TE}=89.5 \mathrm{~ms}$, flip angle $=$ $78^{\circ}, 1.25 \mathrm{~mm}$ isotropic voxel resolution, b-vales $=1000$, $2000,3000 \mathrm{~s} / \mathrm{mm}^{2}, 90$ diffusion weighed volumes for each shell, $18 \mathrm{~b}=0$ volumes). These two scans were taken with opposite phase encoding directions and averaged.

Nathan Kline Institute, Rockland Sample

The Nathan Kline Institute Rockland Sample (NKI) dataset consisted of structural magnetic resonance imaging, resting state functional magnetic resonance imaging data, as well as diffusion magnetic resonance imaging data from 811 subjects (downloaded December 2016 from the INDI S3 Bucket) of a community sample of participants across the lifespan. After excluding subjects based on data and metadata completeness and quality control (see Quality Control), the final subset utilized included 542 subjects $(56 \%$ female, age range $=7-84)$. The study was approved by the Nathan Kline Institute Institutional Review Board and Montclair State University Institutional Review Board and informed consent was obtained from all subjects. A comprehensive description of the imaging parameters can be found online at the NKI website. Briefly, images were collected on a Siemens Magneton Trio with a 12-channel head coil. Subjects underwent one T1-weighted structural scan (TR = $1900 \mathrm{~ms}$, $\mathrm{TE}=2.52 \mathrm{~ms}$, flip angle $=9^{\circ}, 1 \mathrm{~mm}$ isotropic voxel resolution). Subjects underwent three differently parameterized resting state scans, but only one acquisition is used in the present study. The fMRI data was acquired with a gradient-echo planar imaging sequence $(\mathrm{TR}=645$ $\mathrm{ms}, \mathrm{TE}=30 \mathrm{~ms}$, flip angle $=60^{\circ}, 3 \mathrm{~mm}$ isotropic voxel resolution, multiband factor $=4$ ). This resting state run lasted approximately 9:41 seconds, with eyes open and instructions to fixate on a cross. Subjects underwent one diffusion MRI scan $(\mathrm{TR}=2400 \mathrm{~ms}, \mathrm{TE}=85 \mathrm{~ms}$, flip angle $=90^{\circ}, 2 \mathrm{~mm}$ isotropic voxel resolution, $128 \mathrm{diffu}-$ sion weighted volumes, b-value $=1500 \mathrm{~s} / \mathrm{mm}^{2}, 9 \mathrm{~b}=0$ volumes). 


\section{Quality Control}

For HCP, all preprocessed time series were visually inspected from visual artifact. Subject motion measurements during the fMRI and DWI scanning sessions were obtained from the HCP minimal preprocessing pipeline output directories (files: Movement_RelativeRMS.txt and eddy_unwarped_images.eddy_movement_rms). Across fMRI sessions and the single fMRI session, the mean and mean absolute deviation of the motion measurements were calculated, resulting in four summary motion measures per subject. Subjects exceeding 1.5 times the inter-quartile range (in the adverse direction) of the measurement distribution for more than one of these summary motion measurements were excluded. This resulted in the exclusion of four subjects. One additional subject was excluded due to software error during DWI processing.

The NKI was downloaded in December of 2016 from the INDI S3 Bucket. At the time of download, the dataset consisted of 957 T1w (811 subjects), 914 DWI (771 subjects), and 718 fMRI ("acquisition645"; 634 subjects) images. T1w and DWI images, and tractography results were first filtered based on visual inspection. T1w images were filtered based on artifact, such as ringing or ghosting (43 images) and for FreeSurfer reconstruction failure (105 images) as assesses with the ENIGMA QC tools, leaving 809 T1w images (699 subjects). DWI images were filtered based on corrupt data (13 images) and artifact on fitted fractional anisotropy maps (18 images), leaving 883 images (747 subjects). Tractography was run on 781 images (677 subjects) that had both quality controlled T1w and DWI images. Tractography results were filtered based on artifact, which include failure to resolve callosal, cingulum, and/or corticospinal streamlines or errors resulting in visually sparse streamline densities, resulting in 764 tractography runs (661 subjects). T1w, DWI, and fMRI images were then filtered using computed image quality metrics [100 102]. T1w images were excluded if the scan was marked as an outlier (1.5x the inter-quartile range in the adverse direction) in three or more of following quality metric distributions: coefficient of joint variation, contrast-to-noise ratio, signal-to-noise ratio, Dietrich's SNR, FBER, and EFC. DWI images were excluded if the percent of signal outliers, determined by eddy_qc, was greater than 15\%. Furthermore, DWI were excluded if the scan was marked as an outlier (1.5x the inter-quartile range in the adverse direction) in two or more of following quality metric distributions: temporal signal-to-noise ratio, mean voxel intensity outlier count, or max voxel intensity outlier count. fMRI images were excluded if greater than $15 \%$ of time frames exceeded $0.5 \mathrm{~mm}$ framewise displacement. Furthermore, fMRI images were excluded the scan was marked as an outlier (1.5x the inter-quartile range in the adverse direction) in 3 or more of the following quality metric distributions: DVARS standard deviation, DVARS voxelwise standard deviation, temporal signal-to-noise ratio, framewise displacement mean, AFNI's outlier ratio, and AFNI's quality index. This image quality metric filtering excluded zero T1w images, 16 DWI images, and 21 fMRI images. Following this visual and image quality metric filtering, 809 T1w images (699 subjects), 728 DWI images (619 subjects), and 697 fMRI images (633 subjects). The intersection of subjects with at least one valid $\mathrm{T} 1 \mathrm{w}$, DWI, and fMRI images totaled 567 subjects. Finally, age metadata was available for 542 of these subjects.

\section{Image Processing}

Structural, functional, and diffusion images of the HCP dataset were minimally preprocessed according to the description provided in [99. Briefly, T1w images were aligned to MNI space before undergoing FreeSurfer's (version 5.3) cortical reconstruction workflow. fMRI images were corrected for gradient distortion, susceptibility distortion, and motion, and then aligned to the corresponding $\mathrm{T} 1 \mathrm{w}$ with one spline interpolation step. This volume was further corrected for intensity bias and normalized to a mean of 10000. This volume was then projected to the $32 k_{-} f s_{-} L R$ mesh, excluding outliers, and aligned to a common space using a multi-modal surface registration [103. The resultant CIFTI file for each HCP subject used in this study followed the file naming pattern: *_REST $\{1,2\}\}_{-}$LR, RL $\}$Atlas_MSMAll.dtseries.nii.

DWI images were normalized to the mean b0 image, corrected for EPI, eddy current, and gradient non-linearity distortions, and motion, and aligned to subject anatomical space using a boundary-based registration [104. In addition to HCP's minimal preprocessing, diffusion images were corrected for intensity non-uniformity with N4BiasFieldCorrection [105]. FSL's dtifit was used to obtain scalar maps of fractional anisotropy, mean diffusivity, and mean kurtosis. The Dipy toolbox (version 1.1) 106 was used to fit a multi-shell multi-tissue constrained spherical deconvolution [107] to the diffusion data with a spherical harmonics order of 8 , using tissue maps estimated with FSL's fast 108. Tractography was performed using Dipy's Local Tracking module [106]. Multiple instances of probabilistic tractography were run per subject 109, varying the step size and maximum turning angle of the algorithm. Tractography was run at step sizes of $0.25 \mathrm{~mm}, 0.4 \mathrm{~mm}, 0.5 \mathrm{~mm}, 0.6$ $\mathrm{mm}$, and $0.75 \mathrm{~mm}$ with the maximum turning angle set to $20^{\circ}$. Additionally, tractography was run at maximum turning angles of $10^{\circ}, 16^{\circ}, 24^{\circ}$, and $30^{\circ}$ with the step size set to $0.5 \mathrm{~mm}$. For each instance of tractography, streamlines were randomly seeded three times within each voxel of a white matter mask, retained if longer than $10 \mathrm{~mm}$ and with valid endpoints, following Dipy's implementation of anatomically constrained tractography [110], and errant streamlines were filtered based on the cluster confidence index [11].

For NKI, T1w images were submitted to FreeSurfer's 
cortical reconstruction workflow (version 6.0). The FreeSurfer results were used to skull strip the $\mathrm{T} 1 \mathrm{w}$, which was subsequently aligned to MNI space with 6 degrees of freedom. fMRI preprocessing was performed using the fMRIPrep version 1.1.8 [13]. The following description of fMRI preprocessing is based on fMRIPrep's documentation. This workflow utilizes ANTs (2.1.0), FSL (5.0.9), AFNI (16.2.07), FreeSurfer (6.0.1), nipype [112], and nilearn [113. Each T1w was corrected using N4BiasFieldCorrection 105 and skull-stripped using antsBrainExtraction.sh (using the OASIS template). The ANTs derived brain mask was refined with a custom variation of the method to reconcile ANTs-derived and FreeSurfer-derived segmentations of the cortical graymatter of Mindboggle 114. Brain tissue segmentation of cerebrospinal fluid (CSF), white-matter (WM) and gray-matter (GM) was performed on the brain-extracted T1w using fast [108. Functional data was slice time corrected using 3dTshift from AFNI and motion corrected using FSL's mcflirt. "Fieldmap-less" distortion correction was performed by co-registering the functional image to the same-subject T1w with intensity inverted [115] constrained with an average fieldmap template [116], implemented with antsRegistration. This was followed by co-registration to the corresponding $\mathrm{T} 1 \mathrm{w}$ using boundary-based registration [104] with 9 degrees of freedom, using bbregister. Motion correcting transformations, field distortion correcting warp, and BOLD-toT1w transformation warp were concatenated and applied in a single step using antsApplyTransforms using Lanczos interpolation. Frame-wise displacement [117] was calculated for each functional run using the implementation of Nipype. The first four frames of the BOLD data in the T1w space were discarded. Diffusion images were preprocessed following the "DESIGNER" pipeline using MRTrix (3.0) [118, 119], which includes denoising, Gibbs ringing and Rician bias correction, distortion and eddy current correction [120] and B1 field correction. DWI were then aligned to their corresponding $\mathrm{T} 1 \mathrm{w}$ and the MNI space in one interpolation step with B-vectors rotated accordingly. Local models of white matter orientation were estimated in a recursive manner [121 using constrained spherical deconvolution [107] with a spherical harmonics order of 8 . Tractography was performed using Dipy's Local Tracking module 106. Probabilistic streamline tractography was seeded five times in each white matter voxel. Streamlines were propagated with a $0.5 \mathrm{~mm}$ step size and a maximum turning angle set to $20^{\circ}$. Streamlines were retained if longer than $10 \mathrm{~mm}$ and with valid endpoints, following Dipy's implementation of anatomically constrained tractography [110

\section{Network definition}

\section{Parcellation}

As HCP fMRI was provided in $32 k_{-} f s_{-} L R$ space, this data could be parcellated based on the available Schaefer 400 parcellation [40] in the CIFTI file format. For HCP DWI and NKI fMRI and DWI, the Schaefer 400 parcellation was rendered as a volumetric parcellation in each subject's anatomical space within the grey matter ribbon. To transfer the parcellation from fsaverage to subject space, FreeSurfer's mris_ca_label function was used in conjunction with a pre-trained Gaussian classifier surface atlas [122] to register cortical surfaces based on individual curvature and sulcal patterns.

\section{Structural Connectivity}

For HCP, for each tractography instance, streamline counts were normalized by dividing the count between nodes by the geometric average volume of the nodes. Since tractography was run nine times per subject, edge values were collapsed across runs. To do this, the weighted mean was taken with weights based on the proportion of total streamlines at that edge. This amounts to calculating the expected value, where probabilities are based on the proportion of total edge weight across tracotgraphy instances. This operation biases edge weights towards larger values, which reflect tractography instances better parameterized to estimate the geometry of each connection. For NKI, streamline counts were normalized by dividing the count between nodes by the geometric average volume of the nodes.

\section{Functional Connectivity}

For HCP and NKI, each preprocessed BOLD image was linearly detrended, band-pass filtered (0.008-0.08 $\mathrm{Hz}$ ), confound regressed and standardized using Nilearn's signal.clean function, which removes confounds orthogonally to the temporal filters. The confound regression strategy included six motion estimates, mean signal from a white matter, cerebrospinal fluid, and whole brain mask, derivatives of these previous nine regressors, and squares of these 18 terms. Spike regressors were not applied to the HCP data. Spike regressors for frames with motion greater than $0.5 \mathrm{~mm}$ framewise displacement were applied to the NKI data. The 36 parameter strategy (with and without spike regression) has been show to be a relatively effective option to reduce motion-related artifacts [123. Following these preprocessing operations, the mean signal was taken at each node, in either the surface space (HCP) or volumetric anatomical space (NKI).

In the main text, we analyzed data that had been processed using the above procedure. We also performed extensive post-processing of these data to reduce the likeli- 
hood that in-scanner motion contributed to any reported effects [17, 123 125. Specifically, we implemented the following steps for the HCP data:

1. Using the Movement_RelativeRMS .txt time series, we identified frames with motion greater than 0.15 . These frames were immediately censored and not used in the estimation of FC.

2. We also censored any low-motion time points that were within two frames of any of the frames censored in step 1.

3. Following steps 1 and 2, we further censored any sequence of temporally contiguous low-motion frames that was shorter than five frames.

4. Lastly, we excluded a scan if more than $50 \%$ of its frames were flagged as high-motion following steps 1-3, i.e. were censored. If any scan from a given subject was removed, we removed that subject and all of their other scans from analysis.

Given these criteria, we retained 70/95 HCP subjects. Of these remaining subjects, we retained, on average, $90.3 \pm$ $0.97 \%$ of their frames. The frames that were retained had $0.07 \pm 0.01$ relative motion. In contrast, the censored frames had relative motion of $0.15 \pm 0.03$.

Using these data, we fully replicated the results reported in the main text. Specifically, we made predictions of whole-brain FC using the 40 predictors from the 70 low-motion subjects. This resulted in a [40 predictor times 70 subject] matrix of $R^{2}$ values, which we transformed into a $[2800 \times 1]$ vector. We did the same using the data from the main text and computed the similarity (Pearson correlation) between these two vectors. We found a strong correspondence $\left(r=0.997 ; p<10^{-15}\right)$. The findings held at the subject level in which we calculated the similarity of columns $\left(r=0.999 \pm 1.3 \times 10^{-4}\right)$. Next, we performed an analogous procedure at the regional level, resulting in a $[400 \times 40$ predictor $\times 70$ subject] matrix of $R^{2}$ values. Again, we assessed the similarity of the motion-corrected results with those reported in the main text by vectorizing each matrix, transforming them into vectors of $[1120000 \times 1]$, and computing their Pearson correlation. As before, we found an excellent correspondence $(r=0.995)$. We repeated this analysis at the subject level, transforming each of the $70[400 \times$ 40] matrices into $[16000 \times 1]$ vectors and computing separately for each subject, their similarity. Once again, we found a strong correspondence $\left(r=0.9995 \pm 6.13 \times 10^{-4}\right)$. Finally, we modeled FC using pairs of predictors with multilinear models, identified for each region the optimal pair (the one that explained the most variance), and summarized these results with a count matrix, as in the main text. We then computed the correlation of the motion corrected count matrix (its upper triangle) with the matrix reported in the original submission. We found a strong correlation of $r=0.997\left(p<10^{-15}\right)$.
In the case of the NKI data, we performed a similar analysis. Broadly, this strategy involved excluding subjects in the bottom $P \%$ based on their temporal signal to noise ration (TSNR; mean BOLD signal divided by temporal standard deviation [126]) or was in the top $Q \%$ by DVARS (derivative of RMS over voxels; 124]). Of the remaining individuals, we regressed out both variables from their regional $R^{2}$ values and computed their correlation with age. Depending on the values of $P$ and $Q$ different subsets of subjects were retained. We systematically vary the values of $Q$ and $P$ from $5 \%$ to $95 \%$ in increments of $5 \%$ (18 different values). In general, we find broad correspondence between the results reported in the main text and the results at all combinations of parameters. Specifically, the similarity between correlation patterns never fell below a value of $r=0.29$. However, this pattern was obtained using an unreasonable combination of parameters in which only 8 total subjects were retained. For parameter combinations that retained at least half of the full dataset (263 subjects), the minimum similarity was $r=0.83$, with a mean \pm standard deviation of $r=0.89 \pm 0.02$ and range of 0.83 to 0.92 . Over this same range the mean correlation magnitude decreased by $-0.032 \pm 0.005$ while the mean absolute correlation increased by $0.035 \pm 0.003$, suggesting that negative correlations tended to get slightly more negative. Collectively, the results of these two analyses support those reported in the main text.

\section{Predictors \\ Flow graphs}

A flow graph is a transformation of a network's (possibly sparse) connectivity matrix, $A_{i j}$, into a fully-weighted matrix in which the dynamics of a Markov process are embedded into edge weights 41. Flow graphs have been applied in neuroscience for the purposes of community detection [127]. For a continuous time random walk with dynamics $\dot{p}_{i}=-\sum_{j} L_{i j} p_{j}$, the corresponding flow graph is given by $A^{\prime}(t)_{i j}=\left(e^{-t L}\right)_{i} j s_{j}$. In these expressions, the matrix $L$ is the normalized Laplacian whose elements are given by $L_{i j}=D-A / s$, where $s_{i}=\sum_{j} A_{i j}$ is a node's degree or weighted degree and $D$ is the degree diagonal matrix (a square matrix the elements of $s$ along its diagonal). The variable $p_{i}$ represents the probability of finding a random walker on vertex $i$.

The element $A^{\prime}(t)_{i j}$ represents the probabilistic flow of random walkers between nodes $i$ and $j$ at time $t$. Here, we generated flow graphs using both binary and weighted structural connectivity matrices at evaluated them at different Markov times, $t$. Specifically, we focused on $t=1,2.5,5$, and 10 . We refer to these variables as fgbin- or fgwei-followed by Markov time, $t$. 
Navigation

The aim of many networks is to move something from one point in the network to another in as few steps as possible, i.e. to take advantage of shortest paths. However, doing so requires requires full knowledge of a network's shortest path structure, which may not be a realistic assumption, especially for naturally-occurring biological systems like brains. However, it may be the case that simple routing strategies - rules or heuristics for how to move from one node to another - can sometimes uncover optimal or near-optimal shortest paths. One such routing rule is, given a target node $T$, to always move towards the node nearest the target in some metric space, e.g. Euclidean space.

Recently, this navigation approach was applied to brain networks 42. This study defined two novel measures based on navigation of connectome data. First, they defined the number of hops in the shortest path uncovered by the navigation process. We refer to this variable as nav-num. Note that for some node pairs, the navigation procedure leads to a dead end or a cycle in which case the number of hops is listed as $\infty$. For the completed paths, the authors also defined their total length in metric space (in this case Euclidean distance). We refer to this variable as nav-ms and, like nav-num, impute incomplete paths with values of $\infty$.

\section{Communicability}

Communicability [43] is a weighted sum of walks of all lengths between pairs of nodes. For a binary network, it is calculated as $G=e^{A}$ or $\sum_{p=0}^{\infty} \frac{A^{p}}{p !}$. The contribution of direct links (1-step walks) is $\frac{A^{1}}{1 !}$, two-step walks is $\frac{A^{2}}{2 !}$, three-step is $\frac{A^{3}}{3 !}$, and so on. In other words, longer walks have larger denominators and, effectively, are penalized more severely. We denote this measures as comm-bin.

For weighted networks, we follow 44 and first normalize the weighted connectivity matrix as $A^{\prime}=$ $D^{-1 / 2} A D^{-1 / 2}$ where $D$ is the degree diagonal matrix. As before, this normalized matrix is the exponentiated to calculate the weighted communicability $G_{w e i}=e^{A^{\prime}}$. We denote this measures as comm-wei.

\section{Matching Index}

The matching index [26] is a measure of overlap between pairs of nodes based on their connectivity profiles. Suppose $\Gamma_{i}=j: A i j>0$ is the set of all nodes directly connected to node $i$. We can calculate the matching index between nodes $i$ and $j$ as $M_{i j}=\frac{\left|\Gamma_{i \backslash j} \cap \Gamma_{j \backslash i}\right|}{\left|\Gamma_{i \backslash j} \cup \Gamma_{j \backslash i}\right|}$. Here, $\Gamma_{i \backslash j}$ refers to the neighbors of node $i$ excluding node $j$.
Shortest paths

In a network, each edge can be associated with a cost. For binary networks, the cost is identical for each edge; for weighted networks the cost can be obtained by a monotonic transformation of edges' weights to length, e.g. by raising an edge's weight to a negative power. The shortest path between a source node, $s$, and a target node, $t$, is the sequence of edges $\pi_{s \rightarrow t}=$ $\left\{A_{s i}, A_{i j}, \ldots, A_{k t}\right\}$ that minimizes the sum $C_{s i}+C_{i j}+$ $\ldots+C_{k t}$, where $C_{s i}$ is the cost of traversing the edge linking nodes $s$ and $i$.

Here, we calculated shortest paths matrices for the binary network (where the cost is identical for all existing edges) and also for a parameterized affinity-to-cost transformation evaluated at several different parameter values. Specifically, we used the following transformation: $C_{i j}=A_{i j}^{-\gamma}$. We focused on the parameter values $\gamma=0.125,0.25,0.5,1.0,2.0$, and 4.0 . We refer to these measures as pl-bin and pl-wei-followed by $\gamma$ value.

\section{Cosine Similarity}

The cosine similarity measures the angle between two vectors, $x=\left[x_{1}, \ldots, x_{P}\right]$, and $x=\left[y_{1}, \ldots, y_{P}\right]$. Specifically, it measures $S_{x y}=\frac{x \cdot y}{\|x\| \cdot\|y\|}$. Here, we treated regions' connectivity profiles (the row of the connectivity matrix) as vectors and computed the similarity between all pairs of regions. We repeated this procedure for both the binary (cos-bin) and we weighted (cos-wei) connectivity matrices.

\section{Search Information}

Search information measures the amount of information (in bits) required to traverse shortest paths in a network [11, 45]. If the shortest path between nodes $s$ and $t$ is given by $\pi_{s \rightarrow t}=\{s, i, j, \ldots, k, l, t\}$, then the probability of taking that path is given by: $P\left(\pi_{s \rightarrow t}\right)=$ $p_{s i} \times p_{i j} \times \ldots \times p_{k l} \times p_{l t}$, where $p_{i j}=\frac{A_{i j}}{\sum_{j} A_{i j}}$. The information required to take this path, then, is $S\left(\pi_{s \rightarrow t}\right)=$ $\log _{2}\left[P\left(\pi_{s \rightarrow t}\right)\right]$.

Here, we calculated search information based on binary shortest paths (si-bin) and based on shortest paths obtained from each of the weight-to-cost transformations (si-wei- $\gamma$ value).

\section{Mean First Passage Time}

The mean first passage time (MFPT) refers to the expected number of steps a random walk must evolve for a random walked starting at node $i$ to end up at node $j$ [46, 128. Here, we expressed the columns as z-scores 
to remove nodal (column) biases and analyzed the resulting matrices for the binary (mfpt-bin) and weighted (mfpt-wei) connectivity matrices.

\section{Euclidean Distance}

The final predictor that we considered was the Euclidean distance between regional centers of mass $(e u c)$.

\section{Core-periphery analysis}

We used a core-periphery model to analyze the count matrix of how often pairs of predictors were included together in the same multi-linear model. In this context, a core refers to a group of predictor that are densely internally connected and to a periphery, that connect to the core but not to other peripheral predictors [129]. To identify core-periphery structure, we used a variant of a common core-periphery definition in which the transition from core to periphery varies smoothly. Rather than using a binary assignment of nodes to a core or a periphery, this allows nodes to have a graded and continuous assignments. We begin by defining the $N \times 1$ vector $C_{i}$ of non-negative elements [130]. Given this vector, we then defined the matrix $C_{i j}=C_{i} C_{j}$ subject to the constraint that $\sum_{i j} C_{i j}=1$. The values in the vector $C$ are permutations of the vector:

$$
C_{m}^{*}=\frac{1}{1+\exp (-(m-\beta N) \times \tan (\pi \alpha / 2))} .
$$

The coreness of each node is the permutation of $C_{m}^{*}$ that maximizes the core quality function:

$$
R=\sum_{i j} G_{i j} C_{i} C_{j}
$$

This method introduces two free parameters, $\alpha \in[0,1]$ and $\beta \in[0,1]$. The value of $\alpha$ determines the sharpness of the core-periphery boundary. With $\alpha=1$, the transition is binary while the transition with $\alpha=0$ is maximally fuzzy. Similarly, the value of $\beta$ determines the size of the core; as $\beta$ ranges from 0 to 1 , the size of the core varies from $N$ to 0 . In our application, we performed a grid search of 51 logarithmically-spaced values of $\alpha$ and $\beta$, using a simulated annealing algorithm to maximize $R$ (with 25 restarts).

\section{Community detection}

In the main text, we described an analysis in which we clustered brain regions based on the similarity of their optimal predictor. Briefly, this procedure entailed calculating for each brain region the frequency with which predictor, $p$, was optimal, i.e. explained the greatest amount of variance in that regions' FC pattern. This resulted in a vector $\mathbf{h}=\left\{h_{1}, \ldots, h_{p}, \ldots, h_{40}\right\}$ subject to the constraint that $\sum_{p} h_{p}=1$. We then computed the correlation between all pairs of brain regions based on these vectors. We refer to this matrix as $S$, whose element $S_{i j}$ denotes the similarity between feature vectors of regions $i$ and $j$.

To better understand the structure of $S$, we clustered brain regions into communities using modularity maximization [5, 131, 132. To do so, we optimized the modularity quality function:

$$
Q=\sum_{i j} B_{i j} \delta\left(\sigma_{i}, \sigma_{j}\right)
$$

where $B_{i j}=S_{i j}-\gamma \cdot P_{i j}$. In this expression, $P_{i j}$ is the expected weight of the connection between regions $i$ and $j$ and $\gamma$ is a structural resolution parameter that tunes the number of size of detected communities. For simplicity, we set $\gamma=\langle S\rangle=0.296 \approx 0.3$ and used this value for all pairs of brain regions. For completeness, however, we also tested other resolution parameter values, ranging from $\gamma=0$ to $\gamma=1$ in increments of 0.025. We show some of these communities in Fig. S4 $\mathrm{d}$.

We used a generalization of the Louvain algorithm 133 to optimize $Q$. This algorithm is non-deterministic and results in a degeneracy of near-optimal solutions. To resolve this degeneracy, we used a consensus clustering algorithm in which we ran the Louvain algorithm 1000 times (random initial conditions) and computed the coassignment probability for all pairs of brain regions, i.e. the likelihood that they were assigned to the same community [74, 134 136]. Then, we calculated the expected probability that any two nodes were assigned to the same community after randomly and independently permuting the order of each of the 1000 partitions. From these two values, we calculated a new modularity matrix - the observed co-assignment probability minus the expected and clustered this matrix again (repeating the algorithm 1000 times). This sequence - modularity maximization followed by construction of observed and expected coassignment probabilities - was repeated until each of the 1000 runs converged to an identical solution. At this point the consensus algorithm terminated.

\section{DATA AVAILABILITY STATEMENT}

Data from the HCP data can be accessed freely via https://db.humanconnectome.org/ after signing a data use agreement. NKI data is freely available via the following URL: http://fcon_1000.projects. nitrc.org/indi/enhanced/

\section{CODE AVAILABILITY STATEMENT}

Code for estimating the predictors from structural connectivity and using them to predict func- 
tional connectivity is available at https://github.com/ brain-networks/local_scfc.

\section{AUTHOR CONTRIBUTIONS}

FZE and RFB conceived of the study, performed analyses, and generated figures. JF contributed and processed all imaging data. FZE, JF, JS, BM, and RFB wrote and edited the manuscript.

\section{ACKNOWLEDGMENTS}

This material is based upon work supported by the National Science Foundation under Grant No. 07605900003C (RFB; FZE).
[1] Olaf Sporns, Giulio Tononi, and Rolf Kötter, "The human connectome: a structural description of the human brain," PLoS Comput Biol 1, e42 (2005).

[2] Patric Hagmann, Leila Cammoun, Xavier Gigandet, Reto Meuli, Christopher J Honey, Van J Wedeen, and Olaf Sporns, "Mapping the structural core of human cerebral cortex," PLoS Biol 6, e159 (2008).

[3] Olaf Sporns and Jonathan D Zwi, "The small world of the cerebral cortex," Neuroinformatics 2, 145-162 (2004).

[4] Martijn P Van Den Heuvel and Olaf Sporns, "Richclub organization of the human connectome," Journal of Neuroscience 31, 15775-15786 (2011).

[5] Olaf Sporns and Richard F Betzel, "Modular brain networks," Annual review of psychology 67, 613-640 (2016).

[6] Danielle S Bassett, Daniel L Greenfield, Andreas MeyerLindenberg, Daniel R Weinberger, Simon W Moore, and Edward T Bullmore, "Efficient physical embedding of topologically complex information processing networks in brains and computer circuits," PLoS comput biol 6, e1000748 (2010).

[7] Christopher J Honey, Rolf Kötter, Michael Breakspear, and Olaf Sporns, "Network structure of cerebral cortex shapes functional connectivity on multiple time scales," Proceedings of the National Academy of Sciences 104, 10240-10245 (2007).

[8] Christopher J Honey, Olaf Sporns, Leila Cammoun, Xavier Gigandet, Jean-Philippe Thiran, Reto Meuli, and Patric Hagmann, "Predicting human resting-state functional connectivity from structural connectivity," Proceedings of the National Academy of Sciences 106, 2035-2040 (2009).

[9] Ann M Hermundstad, Danielle S Bassett, Kevin S Brown, Elissa M Aminoff, David Clewett, Scott Freeman, Amy Frithsen, Arianne Johnson, Christine M Tipper, Michael B Miller, et al., "Structural foundations of resting-state and task-based functional connectivity in the human brain," Proceedings of the National Academy of Sciences 110, 6169-6174 (2013).

[10] Maxwell H Turner, Kevin Mann, and Thomas R Clandinin, "The connectome predicts resting-state functional connectivity across the drosophila brain," Current Biology (2021).

[11] Joaquín Goñi, Martijn P Van Den Heuvel, Andrea Avena-Koenigsberger, Nieves Velez De Mendizabal, Richard F Betzel, Alessandra Griffa, Patric Hagmann, Bernat Corominas-Murtra, Jean-Philippe Thiran, and Olaf Sporns, "Resting-brain functional connectivity predicted by analytic measures of network communica- tion," Proceedings of the National Academy of Sciences 111, 833-838 (2014).

[12] Andrea Avena-Koenigsberger, Bratislav Misic, and Olaf Sporns, "Communication dynamics in complex brain networks," Nature Reviews Neuroscience 19, 17 (2018).

[13] Oscar Esteban, Christopher J Markiewicz, Ross W Blair, Craig A Moodie, A Ilkay Isik, Asier Erramuzpe, James D Kent, Mathias Goncalves, Elizabeth DuPre, Madeleine Snyder, et al., "fmriprep: a robust preprocessing pipeline for functional mri," Nature methods 16, 111-116 (2019).

[14] Matthew Cieslak, Philip A Cook, Xiaosong He, FangCheng Yeh, Thijs Dhollander, Azeez Adebimpe, Geoffrey K Aguirre, Danielle S Bassett, Richard F Betzel, Josiane Bourque, et al., "Qsiprep: An integrative platform for preprocessing and reconstructing diffusion mri," bioRxiv (2020).

[15] Kanika Bansal, Johan Nakuci, and Sarah Feldt Muldoon, "Personalized brain network models for assessing structure-function relationships," Current Opinion in Neurobiology 52, 42-47 (2018).

[16] Laura E Suárez, Ross D Markello, Richard F Betzel, and Bratislav Misic, "Linking structure and function in macroscale brain networks," Trends in Cognitive Sciences (2020).

[17] Yusuke Adachi, Takahiro Osada, Olaf Sporns, Takamitsu Watanabe, Teppei Matsui, Kentaro Miyamoto, and Yasushi Miyashita, "Functional connectivity between anatomically unconnected areas is shaped by collective network-level effects in the macaque cortex," Cerebral cortex 22, 1586-1592 (2012).

[18] Paula Sanz-Leon, Stuart A Knock, Andreas Spiegler, and Viktor K Jirsa, "Mathematical framework for largescale brain network modeling in the virtual brain," Neuroimage 111, 385-430 (2015).

[19] Jessica S Damoiseaux and Michael D Greicius, "Greater than the sum of its parts: a review of studies combining structural connectivity and resting-state functional connectivity," Brain structure and function 213, 525533 (2009).

[20] Bratislav Mišić, Richard F Betzel, Marcel A De Reus, Martijn P Van Den Heuvel, Marc G Berman, Anthony R McIntosh, and Olaf Sporns, "Network-level structurefunction relationships in human neocortex," Cerebral Cortex 26, 3285-3296 (2016).

[21] Bratislav Mišić, Richard F Betzel, Azadeh Nematzadeh, Joaquin Goni, Alessandra Griffa, Patric Hagmann, Alessandro Flammini, Yong-Yeol Ahn, and Olaf 
Sporns, "Cooperative and competitive spreading dynamics on the human connectome," Neuron 86, 15181529 (2015).

[22] Andrea Avena-Koenigsberger, Xiaoran Yan, Artemy Kolchinsky, Martijn van den Heuvel, Patric Hagmann, and Olaf Sporns, "A spectrum of routing strategies for brain networks," PLoS computational biology 15, e1006833 (2019).

[23] Richard F Betzel, John D Medaglia, Ari E Kahn, Jonathan Soffer, Daniel R Schonhaut, and Danielle S Bassett, "Structural, geometric and genetic factors predict interregional brain connectivity patterns probed by electrocorticography," Nature biomedical engineering $\mathbf{3}$, 902-916 (2019).

[24] Arnaud Messé, David Rudrauf, Alain Giron, and Guillaume Marrelec, "Predicting functional connectivity from structural connectivity via computational models using mri: an extensive comparison study," NeuroImage 111, 65-75 (2015).

[25] Marian Boguna, Dmitri Krioukov, and Kimberly C Claffy, "Navigability of complex networks," Nature Physics 5, 74-80 (2009).

[26] Claus-C Hilgetag, Gully APC Burns, Marc A O'Neill, Jack W Scannell, and Malcolm P Young, "Anatomical connectivity defines the organization of clusters of cortical areas in the macaque and the cat," Philosophical Transactions of the Royal Society of London. Series B: Biological Sciences 355, 91-110 (2000).

[27] Caio Seguin, Ye Tian, and Andrew Zalesky, "Network communication models improve the behavioral and functional predictive utility of the human structural connectome," Network Neuroscience 4, 980-1006 (2020).

[28] Zijin Gu, Keith Wakefield Jamison, Mert Rory Sabuncu, and Amy Kuceyeski, "Regional structural-functional connectome coupling is heritable and associated with age, sex and cognition in adults," bioRxiv (2020).

[29] Alessandra Griffa, Enrico Amico, Raphael Liegeois, Dimitri Van De Ville, and Maria Giulia Preti, "Structure-function interplay as signature for brain decoding and fingerprinting," bioRxiv (2021).

[30] Graham L Baum, Zaixu Cui, David R Roalf, Rastko Ciric, Richard F Betzel, Bart Larsen, Matthew Cieslak, Philip A Cook, Cedric H Xia, Tyler M Moore, et al., "Development of structure-function coupling in human brain networks during youth," Proceedings of the National Academy of Sciences 117, 771-778 (2020).

[31] Bertha Vázquez-Rodríguez, Laura E Suárez, Ross D Markello, Golia Shafiei, Casey Paquola, Patric Hagmann, Martijn P Van Den Heuvel, Boris C Bernhardt, R Nathan Spreng, and Bratislav Misic, "Gradients of structure-function tethering across neocortex," Proceedings of the National Academy of Sciences 116, 21219-21227 (2019).

[32] Richard F Betzel, Lisa Byrge, Ye He, Joaquín Goñi, Xi-Nian Zuo, and Olaf Sporns, "Changes in structural and functional connectivity among resting-state networks across the human lifespan," Neuroimage 102, 345-357 (2014).

[33] Catherine Lebel, Lindsay Walker, Alexander Leemans, Linda Phillips, and Christian Beaulieu, "Microstructural maturation of the human brain from childhood to adulthood," Neuroimage 40, 1044-1055 (2008).
[34] Joshua Faskowitz, Xiaoran Yan, Xi-Nian Zuo, and Olaf Sporns, "Weighted stochastic block models of the human connectome across the life span," Scientific reports 8, 1-16 (2018).

[35] Linda Geerligs, Remco J Renken, Emi Saliasi, Natasha M Maurits, and Monicque M Lorist, "A brainwide study of age-related changes in functional connectivity," Cerebral cortex 25, 1987-1999 (2015).

[36] Micaela Y Chan, Denise C Park, Neil K Savalia, Steven E Petersen, and Gagan S Wig, "Decreased segregation of brain systems across the healthy adult lifespan," Proceedings of the National Academy of Sciences 111, E4997-E5006 (2014).

[37] Xi-Nian Zuo, Ye He, Richard F Betzel, Stan Colcombe, Olaf Sporns, and Michael P Milham, "Human connectomics across the life span," Trends in cognitive sciences 21, 32-45 (2017).

[38] David C Van Essen, Stephen M Smith, Deanna M Barch, Timothy EJ Behrens, Essa Yacoub, Kamil Ugurbil, Wu-Minn HCP Consortium, et al., "The wu-minn human connectome project: an overview," Neuroimage 80, 62-79 (2013).

[39] Kate Brody Nooner, Stanley Colcombe, Russell Tobe, Maarten Mennes, Melissa Benedict, Alexis Moreno, Laura Panek, Shaquanna Brown, Stephen Zavitz, Qingyang Li, et al., "The nki-rockland sample: a model for accelerating the pace of discovery science in psychiatry," Frontiers in neuroscience 6, 152 (2012).

[40] Alexander Schaefer, Ru Kong, Evan M Gordon, Timothy O Laumann, Xi-Nian Zuo, Avram J Holmes, Simon B Eickhoff, and BT Thomas Yeo, "Local-global parcellation of the human cerebral cortex from intrinsic functional connectivity mri," Cerebral cortex 28, 30953114 (2018).

[41] Renaud Lambiotte, Roberta Sinatra, J-C Delvenne, Tim S Evans, Mauricio Barahona, and Vito Latora, "Flow graphs: Interweaving dynamics and structure," Physical Review E 84, 017102 (2011).

[42] Caio Seguin, Martijn P Van Den Heuvel, and Andrew Zalesky, "Navigation of brain networks," Proceedings of the National Academy of Sciences 115, 6297-6302 (2018).

[43] Ernesto Estrada and Naomichi Hatano, "Communicability in complex networks," Physical Review E 77, 036111 (2008).

[44] Jonathan J Crofts and Desmond J Higham, "A weighted communicability measure applied to complex brain networks," Journal of the Royal Society Interface 6, 411414 (2009).

[45] Martin Rosvall, Andreas Grönlund, Petter Minnhagen, and Kim Sneppen, "Searchability of networks," Physical Review E 72, 046117 (2005).

[46] Jae Dong Noh and Heiko Rieger, "Random walks on complex networks," Physical review letters 92, 118701 (2004).

[47] Ross D Markello and Bratislav Misic, "Comparing spatial null models for brain maps," NeuroImage , 118052 (2021).

[48] Yusuf Osmanlığlu, Birkan Tunç, Drew Parker, Mark A Elliott, Graham L Baum, Rastko Ciric, Theodore D Satterthwaite, Raquel E Gur, Ruben C Gur, and Ragini Verma, "System-level matching of structural and functional connectomes in the human brain," NeuroImage 199, 93-104 (2019). 
[49] František Váša, Jakob Seidlitz, Rafael Romero-Garcia, Kirstie J Whitaker, Gideon Rosenthal, Petra E Vértes, Maxwell Shinn, Aaron Alexander-Bloch, Peter Fonagy, Raymond J Dolan, et al., "Adolescent tuning of association cortex in human structural brain networks," Cerebral Cortex 28, 281-294 (2018).

[50] Mark W Woolrich and Klaas E Stephan, "Biophysical network models and the human connectome," Neuroimage 80, 330-338 (2013).

[51] Peng Wang, Ru Kong, Xiaolu Kong, Raphaël Liégeois, Csaba Orban, Gustavo Deco, Martijn P Van Den Heuvel, and BT Thomas Yeo, "Inversion of a large-scale circuit model reveals a cortical hierarchy in the dynamic resting human brain," Science advances $\mathbf{5}$, eaat7854 (2019).

[52] Rishidev Chaudhuri, Kenneth Knoblauch, Marie-Alice Gariel, Henry Kennedy, and Xiao-Jing Wang, "A largescale circuit mechanism for hierarchical dynamical processing in the primate cortex," Neuron 88, 419-431 (2015).

[53] Xiaolu Kong, Ru Kong, Csaba Orban, Wang Peng, Shaoshi Zhang, Kevin Anderson, Avram Holmes, John D Murray, Gustavo Deco, Martijn van den Heuvel, et al., "Anatomical and functional gradients shape dynamic functional connectivity in the human brain," bioRxiv (2021).

[54] Justin L Vincent, Gaurav H Patel, Michael D Fox, Abraham Z Snyder, Justin T Baker, David C Van Essen, John M Zempel, Lawrence H Snyder, Maurizio Corbetta, and Marcus E Raichle, "Intrinsic functional architecture in the anaesthetized monkey brain," Nature 447, 83-86 (2007).

[55] Longwen Huang, Justus M Kebschull, Daniel Fürth, Simon Musall, Matthew T Kaufman, Anne K Churchland, and Anthony M Zador, "Bricseq bridges brain-wide interregional connectivity to neural activity and gene expression in single animals," Cell 182, 177-188 (2020).

[56] Randy L Buckner and Fenna M Krienen, "The evolution of distributed association networks in the human brain," Trends in cognitive sciences 17, 648-665 (2013).

[57] Jason Hill, Terrie Inder, Jeffrey Neil, Donna Dierker, John Harwell, and David Van Essen, "Similar patterns of cortical expansion during human development and evolution," Proceedings of the National Academy of Sciences 107, 13135-13140 (2010).

[58] Gustavo Deco, Viktor K Jirsa, Peter A Robinson, Michael Breakspear, and Karl Friston, "The dynamic brain: from spiking neurons to neural masses and cortical fields," PLoS computational biology 4, e1000092 (2008).

[59] Gustavo Deco, Viktor K Jirsa, and Anthony R McIntosh, "Emerging concepts for the dynamical organization of resting-state activity in the brain," Nature Reviews Neuroscience 12, 43-56 (2011).

[60] Petra Ritter, Michael Schirner, Anthony R McIntosh, and Viktor K Jirsa, "The virtual brain integrates computational modeling and multimodal neuroimaging," Brain connectivity 3, 121-145 (2013).

[61] Zhen-Qi Liu, Bertha Vazquez-Rodriguez, R Nathan Spreng, Boris Bernhardt, Richard F Betzel, and Bratislav Misic, "Time-resolved structure-function coupling in brain networks," bioRxiv (2021).

[62] Raphaël Liégeois, Erik Ziegler, Christophe Phillips, Pierre Geurts, Francisco Gómez, Mohamed Ali Bahri,
BT Thomas Yeo, Andrea Soddu, Audrey Vanhaudenhuyse, Steven Laureys, et al., "Cerebral functional connectivity periodically (de) synchronizes with anatomical constraints," Brain structure and function 221, 29852997 (2016).

[63] Makoto Fukushima, Richard F Betzel, Ye He, Martijn $P$ van den Heuvel, Xi-Nian Zuo, and Olaf Sporns, "Structure-function relationships during segregated and integrated network states of human brain functional connectivity," Brain Structure and Function 223, 1091-1106 (2018).

[64] Christopher J Honey, Jean-Philippe Thivierge, and Olaf Sporns, "Can structure predict function in the human brain?" Neuroimage 52, 766-776 (2010).

[65] Paula Sanz Leon, Stuart A Knock, M Marmaduke Woodman, Lia Domide, Jochen Mersmann, Anthony R McIntosh, and Viktor Jirsa, "The virtual brain: a simulator of primate brain network dynamics," Frontiers in neuroinformatics 7, 10 (2013).

[66] Karla Batista-García-Ramó and Caridad Ivette Fernández-Verdecia, "What we know about the brain structure-function relationship," Behavioral Sciences 8, 39 (2018).

[67] T Sarwar, Y Tian, BTT Yeo, K Ramamohanarao, and A Zalesky, "Structure-function coupling in the human connectome: A machine learning approach," NeuroImage 226, 117609 (2021).

[68] Christopher W Lynn and Danielle S Bassett, "The physics of brain network structure, function and control," Nature Reviews Physics 1, 318-332 (2019).

[69] Cassiano O Becker, Sérgio Pequito, George J Pappas, Michael B Miller, Scott T Grafton, Danielle S Bassett, and Victor M Preciado, "Spectral mapping of brain functional connectivity from diffusion imaging," Scientific reports 8, 1-15 (2018).

[70] Gideon Rosenthal, František Váša, Alessandra Griffa, Patric Hagmann, Enrico Amico, Joaquín Goñi, Galia Avidan, and Olaf Sporns, "Mapping higher-order relations between brain structure and function with embedded vector representations of connectomes," Nature communications 9, 1-12 (2018).

[71] Aaron F Alexander-Bloch, Petra E Vértes, Reva Stidd, François Lalonde, Liv Clasen, Judith Rapoport, Jay Giedd, Edward T Bullmore, and Nitin Gogtay, "The anatomical distance of functional connections predicts brain network topology in health and schizophrenia," Cerebral cortex 23, 127-138 (2013).

[72] Martijn P Van Den Heuvel, Cornelis J Stam, René S Kahn, and Hilleke E Hulshoff Pol, "Efficiency of functional brain networks and intellectual performance," Journal of Neuroscience 29, 7619-7624 (2009).

[73] Vito Latora and Massimo Marchiori, "Efficient behavior of small-world networks," Physical review letters 87, 198701 (2001).

[74] Richard F Betzel and Danielle S Bassett, "Specificity and robustness of long-distance connections in weighted, interareal connectomes," Proceedings of the National Academy of Sciences 115, E4880-E4889 (2018).

[75] Ashish Raj and Fon Powell, "Models of network spread and network degeneration in brain disorders," Biological Psychiatry: Cognitive Neuroscience and Neuroimaging 3, 788-797 (2018).

[76] Ying-Qiu Zheng, Yu Zhang, Yvonne Yau, Yashar Zeighami, Kevin Larcher, Bratislav Misic, and Alain 
Dagher, "Local vulnerability and global connectivity jointly shape neurodegenerative disease propagation," PLoS biology 17, e3000495 (2019).

[77] Antoine Allard and M Ángeles Serrano, "Navigable maps of structural brain networks across species," PLoS computational biology 16, e1007584 (2020).

[78] Jennifer Stiso and Danielle S Bassett, "Spatial embedding imposes constraints on neuronal network architectures," Trends in cognitive sciences 22, 1127-1142 (2018).

[79] Marta Rivera-Alba, Shiv N Vitaladevuni, Yuriy Mishchenko, Zhiyuan Lu, Shin-ya Takemura, Lou Scheffer, Ian A Meinertzhagen, Dmitri B Chklovskii, and Gonzalo G de Polavieja, "Wiring economy and volume exclusion determine neuronal placement in the drosophila brain," Current Biology 21, 2000-2005 (2011).

[80] Mária Ercsey-Ravasz, Nikola T Markov, Camille Lamy, David C Van Essen, Kenneth Knoblauch, Zoltán Toroczkai, and Henry Kennedy, "A predictive network model of cerebral cortical connectivity based on a distance rule," Neuron 80, 184-197 (2013).

[81] David Samu, Anil K Seth, and Thomas Nowotny, "Influence of wiring cost on the large-scale architecture of human cortical connectivity," PLoS Comput Biol 10, e1003557 (2014).

[82] Farnaz Zamani Esfahlani, Maxwell A Bertolero, Danielle S Bassett, and Richard F Betzel, "Spaceindependent community and hub structure of functional brain networks," NeuroImage 211, 116612 (2020).

[83] Sabine Oligschläger, Julia M Huntenburg, Johannes Golchert, Mark E Lauckner, Tyler Bonnen, and Daniel S Margulies, "Gradients of connectivity distance are anchored in primary cortex," Brain Structure and Function 222, 2173-2182 (2017).

[84] Sabine Oligschläger, Ting Xu, Blazej M Baczkowski, Marcel Falkiewicz, Arnaud Falchier, Gary Linn, and Daniel S Margulies, "Gradients of connectivity distance in the cerebral cortex of the macaque monkey," Brain Structure and Function 224, 925-935 (2019).

[85] Roberto Cabeza, Nicole D Anderson, Jill K Locantore, and Anthony R McIntosh, "Aging gracefully: compensatory brain activity in high-performing older adults," Neuroimage 17, 1394-1402 (2002).

[86] Maria Grazia Puxeddu, Joshua Faskowitz, Richard F Betzel, Manuela Petti, Laura Astolfi, and Olaf Sporns, "The modular organization of brain cortical connectivity across the human lifespan," NeuroImage 218, 116974 (2020).

[87] Linda Geerligs, Kamen A Tsvetanov, and Richard N Henson, "Challenges in measuring individual differences in functional connectivity using fmri: the case of healthy aging," Human brain mapping 38, 4125-4156 (2017).

[88] Linda Geerligs, Mikail Rubinov, Richard N Henson, et al., "State and trait components of functional connectivity: individual differences vary with mental state," Journal of Neuroscience 35, 13949-13961 (2015).

[89] Clarisse I Mark, Erin L Mazerolle, and J Jean Chen, "Metabolic and vascular origins of the bold effect: Implications for imaging pathology and resting-state brain function," Journal of Magnetic Resonance Imaging 42, 231-246 (2015).
[90] Daniel A Handwerker, John M Ollinger, and Mark D'Esposito, "Variation of bold hemodynamic responses across subjects and brain regions and their effects on statistical analyses," Neuroimage 21, 1639-1651 (2004).

[91] Daniel A Handwerker, Javier Gonzalez-Castillo, Mark D'esposito, and Peter A Bandettini, "The continuing challenge of understanding and modeling hemodynamic variation in fmri," Neuroimage 62, 1017-1023 (2012).

[92] Daniel A Handwerker, Adam Gazzaley, Ben A Inglis, and Mark D'Esposito, "Reducing vascular variability of fmri data across aging populations using a breathholding task," Human brain mapping 28, 846-859 (2007).

[93] Francois Rheault, Philippe Poulin, Alex Valcourt Caron, Etienne St-Onge, and Maxime Descoteaux, "Common misconceptions, hidden biases and modern challenges of dmri tractography," Journal of neural engineering 17, 011001 (2020).

[94] Cibu Thomas, Q Ye Frank, M Okan Irfanoglu, Pooja Modi, Kadharbatcha S Saleem, David A Leopold, and Carlo Pierpaoli, "Anatomical accuracy of brain connections derived from diffusion mri tractography is inherently limited," Proceedings of the National Academy of Sciences 111, 16574-16579 (2014).

[95] Colin Reveley, Anil K Seth, Carlo Pierpaoli, Afonso C Silva, David Yu, Richard C Saunders, David A Leopold, and Q Ye Frank, "Superficial white matter fiber systems impede detection of long-range cortical connections in diffusion mr tractography," Proceedings of the National Academy of Sciences 112, E2820-E2828 (2015).

[96] Maedbh King, Carlos R Hernandez-Castillo, Russell A Poldrack, Richard B Ivry, and Jörn Diedrichsen, "Functional boundaries in the human cerebellum revealed by a multi-domain task battery," Nature neuroscience 22, 1371-1378 (2019).

[97] Gidon Levakov, Joshua Faskowitz, Galia Avidan, and Olaf Sporns, "Mapping structure to function and behavior with individual-level connectome embedding," bioRxiv (2021).

[98] Timothy S Coalson, David C Van Essen, and Matthew F Glasser, "The impact of traditional neuroimaging methods on the spatial localization of cortical areas," Proceedings of the National Academy of Sciences 115, E6356-E6365 (2018).

[99] Matthew F Glasser, Stamatios N Sotiropoulos, J Anthony Wilson, Timothy S Coalson, Bruce Fischl, Jesper L Andersson, Junqian Xu, Saad Jbabdi, Matthew Webster, Jonathan R Polimeni, et al., "The minimal preprocessing pipelines for the human connectome project," Neuroimage 80, 105-124 (2013).

[100] Oscar Esteban, Daniel Birman, Marie Schaer, Oluwasanmi O Koyejo, Russell A Poldrack, and Krzysztof J Gorgolewski, "Mriqc: Advancing the automatic prediction of image quality in mri from unseen sites," PloS one 12, e0184661 (2017).

[101] David R Roalf, Megan Quarmley, Mark A Elliott, Theodore D Satterthwaite, Simon N Vandekar, Kosha Ruparel, Efstathios D Gennatas, Monica E Calkins, Tyler M Moore, Ryan Hopson, et al., "The impact of quality assurance assessment on diffusion tensor imaging outcomes in a large-scale population-based cohort," Neuroimage 125, 903-919 (2016).

[102] Matteo Bastiani, Michiel Cottaar, Sean P Fitzgibbon, Sana Suri, Fidel Alfaro-Almagro, Stamatios N Sotiropoulos, Saad Jbabdi, and Jesper LR Anders- 
son, "Automated quality control for within and between studies diffusion mri data using a non-parametric framework for movement and distortion correction," Neuroimage 184, 801-812 (2019).

[103] Emma C Robinson, Saad Jbabdi, Matthew F Glasser, Jesper Andersson, Gregory C Burgess, Michael P Harms, Stephen M Smith, David C Van Essen, and Mark Jenkinson, "Msm: a new flexible framework for multimodal surface matching," Neuroimage 100, 414426 (2014).

[104] Douglas N Greve and Bruce Fischl, "Accurate and robust brain image alignment using boundary-based registration," Neuroimage 48, 63-72 (2009).

[105] Nicholas J Tustison, Brian B Avants, Philip A Cook, Yuanjie Zheng, Alexander Egan, Paul A Yushkevich, and James C Gee, "N4itk: improved n3 bias correction," IEEE transactions on medical imaging 29, 1310-1320 (2010).

[106] Eleftherios Garyfallidis, Matthew Brett, Bagrat Amirbekian, Ariel Rokem, Stefan Van Der Walt, Maxime Descoteaux, and Ian Nimmo-Smith, "Dipy, a library for the analysis of diffusion mri data," Frontiers in neuroinformatics $\mathbf{8}, 8$ (2014).

[107] J-Donald Tournier, Fernando Calamante, and Alan Connelly, "Robust determination of the fibre orientation distribution in diffusion mri: non-negativity constrained super-resolved spherical deconvolution," Neuroimage 35, 1459-1472 (2007).

[108] Yongyue Zhang, Michael Brady, and Stephen Smith, "Segmentation of brain $\mathrm{mr}$ images through a hidden markov random field model and the expectationmaximization algorithm," IEEE transactions on medical imaging 20, 45-57 (2001).

[109] Hiromasa Takemura, Cesar F Caiafa, Brian A Wandell, and Franco Pestilli, "Ensemble tractography," PLoS computational biology 12, e1004692 (2016).

[110] Robert E Smith, Jacques-Donald Tournier, Fernando Calamante, and Alan Connelly, "Anatomicallyconstrained tractography: improved diffusion mri streamlines tractography through effective use of anatomical information," Neuroimage 62, 1924-1938 (2012).

[111] Kesshi M Jordan, Bagrat Amirbekian, Anisha Keshavan, and Roland G Henry, "Cluster confidence index: a streamline-wise pathway reproducibility metric for diffusion-weighted mri tractography," Journal of Neuroimaging 28, 64-69 (2018).

[112] Krzysztof Gorgolewski, Christopher D Burns, Cindee Madison, Dav Clark, Yaroslav O Halchenko, Michael L Waskom, and Satrajit S Ghosh, "Nipype: a flexible, lightweight and extensible neuroimaging data processing framework in python," Frontiers in neuroinformatics 5, 13 (2011).

[113] Alexandre Abraham, Fabian Pedregosa, Michael Eickenberg, Philippe Gervais, Andreas Mueller, Jean Kossaifi, Alexandre Gramfort, Bertrand Thirion, and Gaël Varoquaux, "Machine learning for neuroimaging with scikit-learn," Frontiers in neuroinformatics 8, 14 (2014).

[114] Arno Klein, Satrajit S Ghosh, Forrest S Bao, Joachim Giard, Yrjö Häme, Eliezer Stavsky, Noah Lee, Brian Rossa, Martin Reuter, Elias Chaibub Neto, et al., "Mindboggling morphometry of human brains," PLoS computational biology 13, e1005350 (2017).
[115] Sijia Wang, Daniel J Peterson, J Christopher Gatenby, Wenbin Li, Thomas J Grabowski, and Tara M Madhyastha, "Evaluation of field map and nonlinear registration methods for correction of susceptibility artifacts in diffusion mri," Frontiers in neuroinformatics 11, 17 (2017).

[116] Jeffrey Mark Treiber, Nathan S White, Tyler Christian Steed, Hauke Bartsch, Dominic Holland, Nikdokht Farid, Carrie R McDonald, Bob S Carter, Anders Martin Dale, and Clark C Chen, "Characterization and correction of geometric distortions in 814 diffusion weighted images," PloS one 11, e0152472 (2016).

[117] Jonathan D Power, Anish Mitra, Timothy O Laumann, Abraham Z Snyder, Bradley L Schlaggar, and Steven E Petersen, "Methods to detect, characterize, and remove motion artifact in resting state fmri," Neuroimage 84, 320-341 (2014).

[118] Benjamin Ades-Aron, Jelle Veraart, Peter Kochunov, Stephen McGuire, Paul Sherman, Elias Kellner, Dmitry S Novikov, and Els Fieremans, "Evaluation of the accuracy and precision of the diffusion parameter estimation with gibbs and noise removal pipeline," NeuroImage 183, 532-543 (2018).

[119] J-Donald Tournier, Robert Smith, David Raffelt, Rami Tabbara, Thijs Dhollander, Maximilian Pietsch, Daan Christiaens, Ben Jeurissen, Chun-Hung Yeh, and Alan Connelly, "Mrtrix3: A fast, flexible and open software framework for medical image processing and visualisation," NeuroImage 202, 116137 (2019).

[120] Jesper LR Andersson and Stamatios N Sotiropoulos, "An integrated approach to correction for off-resonance effects and subject movement in diffusion mr imaging," Neuroimage 125, 1063-1078 (2016).

[121] Chantal MW Tax, Ben Jeurissen, Sjoerd B Vos, Max A Viergever, and Alexander Leemans, "Recursive calibration of the fiber response function for spherical deconvolution of diffusion mri data," Neuroimage 86, 67-80 (2014).

[122] Bruce Fischl, André Van Der Kouwe, Christophe Destrieux, Eric Halgren, Florent Ségonne, David H Salat, Evelina Busa, Larry J Seidman, Jill Goldstein, David Kennedy, et al., "Automatically parcellating the human cerebral cortex," Cerebral cortex 14, 11-22 (2004).

[123] Linden Parkes, Ben Fulcher, Murat Yücel, and Alex Fornito, "An evaluation of the efficacy, reliability, and sensitivity of motion correction strategies for restingstate functional mri," Neuroimage 171, 415-436 (2018).

[124] Jonathan D Power, Kelly A Barnes, Abraham Z Snyder, Bradley L Schlaggar, and Steven E Petersen, "Spurious but systematic correlations in functional connectivity mri networks arise from subject motion," Neuroimage 59, 2142-2154 (2012).

[125] Rastko Ciric, Daniel H Wolf, Jonathan D Power, David R Roalf, Graham L Baum, Kosha Ruparel, Russell T Shinohara, Mark A Elliott, Simon B Eickhoff, Christos Davatzikos, et al., "Benchmarking of participant-level confound regression strategies for the control of motion artifact in studies of functional connectivity," Neuroimage 154, 174-187 (2017).

[126] Gunnar Krüger and Gary H Glover, "Physiological noise in oxygenation-sensitive magnetic resonance imaging," Magnetic Resonance in Medicine: An Official Journal of the International Society for Magnetic Resonance in Medicine 46, 631-637 (2001). 
[127] Richard F Betzel, Alessandra Griffa, Andrea AvenaKoenigsberger, Joaquín Goñi, Jean-Philippe Thiran, Patric Hagmann, and Olaf Sporns, "Multi-scale community organization of the human structural connectome and its relationship with resting-state functional connectivity," Network Science 1, 353-373 (2013).

[128] Joaquín Goñi, Andrea Avena-Koenigsberger, Nieves Velez de Mendizabal, Martijn $\mathrm{P}$ van den Heuvel, Richard F Betzel, and Olaf Sporns, "Exploring the morphospace of communication efficiency in complex networks," PLoS One 8, e58070 (2013).

[129] Stephen P Borgatti and Martin G Everett, "Models of core/periphery structures," Social networks 21, 375-395 (2000).

[130] Puck Rombach, Mason A Porter, James H Fowler, and Peter J Mucha, "Core-periphery structure in networks (revisited)," SIAM review 59, 619-646 (2017).

[131] Mark EJ Newman and Michelle Girvan, "Finding and evaluating community structure in networks," Physical review E 69, 026113 (2004).
[132] Vincent A Traag, Paul Van Dooren, and Yurii Nesterov, "Narrow scope for resolution-limit-free community detection," Physical Review E 84, 016114 (2011).

[133] Inderjit S Jutla, Lucas GS Jeub, and Peter J Mucha, "A generalized louvain method for community detection implemented in matlab," URL http://netwiki. amath. unc. edu/GenLouvain (2011).

[134] Richard F Betzel, Maxwell A Bertolero, Evan M Gordon, Caterina Gratton, Nico UF Dosenbach, and Danielle S Bassett, "The community structure of functional brain networks exhibits scale-specific patterns of inter-and intra-subject variability," Neuroimage 202, 115990 (2019).

[135] Richard F Betzel, Lisa Byrge, Farnaz Zamani Esfahlani, and Daniel P Kennedy, "Temporal fluctuations in the brain's modular architecture during movie-watching," NeuroImage , 116687 (2020).

[136] Richard F Betzel, "Organizing principles of whole-brain functional connectivity in zebrafish larvae," Network Neuroscience 4, 234-256 (2020). 


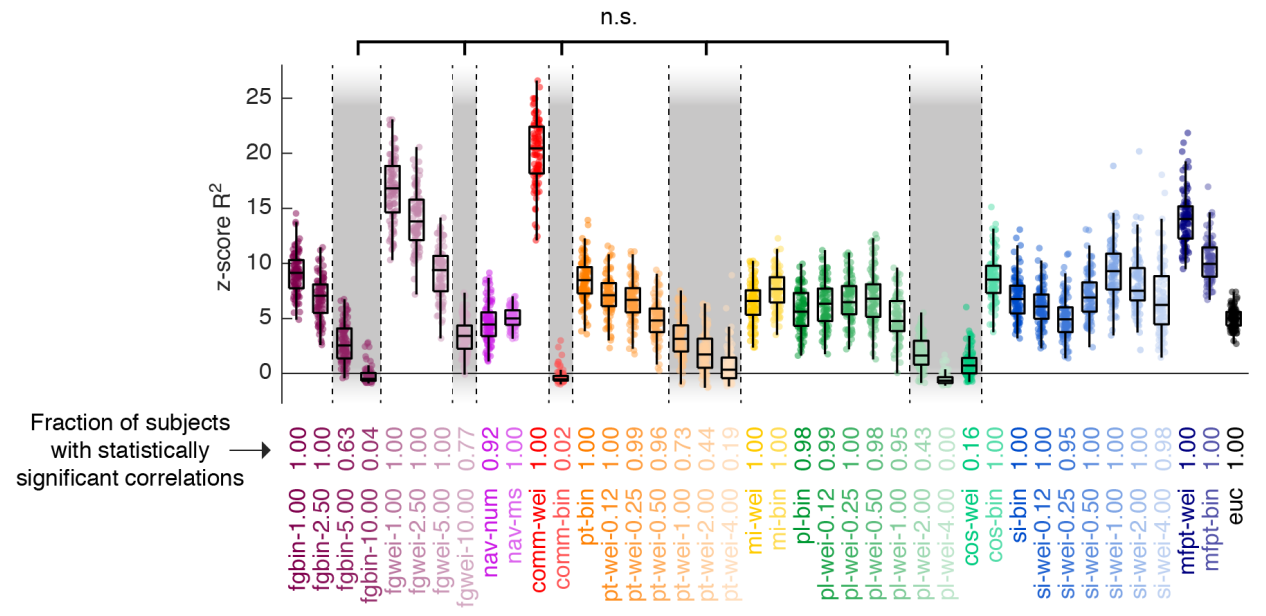

FIG. S1. Statistical analysis of global structure-function coupling. In the main text we reported structure-function coupling at the global (whole-brain) level. Here, we use spin tests to assess the statistical significance of the variance explained. Specifically, we calculated the observed structure-function coupling magnitude $\left(R^{2}\right)$ for each subject and predictor. We also obtained 1000 null values of the same measure using the spin test procedure described in the main text. Using these null values, we z-scored each observed $R^{2}$ measure. This procedure results in 95 z-scores for each of the 40 predictors. We evaluated these data in two ways. First, we transformed the z-scores into p-values and calculated for each predictor the fraction of subjects that exhibited a statistically significant correlation (corrected for multiple comparisons by fixing the false discovery rate at $5 \%$ and adjusting the critical p-value; $\left.p_{\text {adjusted }}=0.0208\right)$. In addition, we identified predictors whose distribution of $\mathrm{z}$-scores fully excluded a value of 0 . In this figure, we show for each predictor the distribution of z-scores. Gray rectangles around predictors identify those whose distribution included a value of zero and were considered "not significant". The row of numbers below the boxplot indicates, for each predictor, the fraction of subjects that exhibited a statistically significant structure-function correlation.
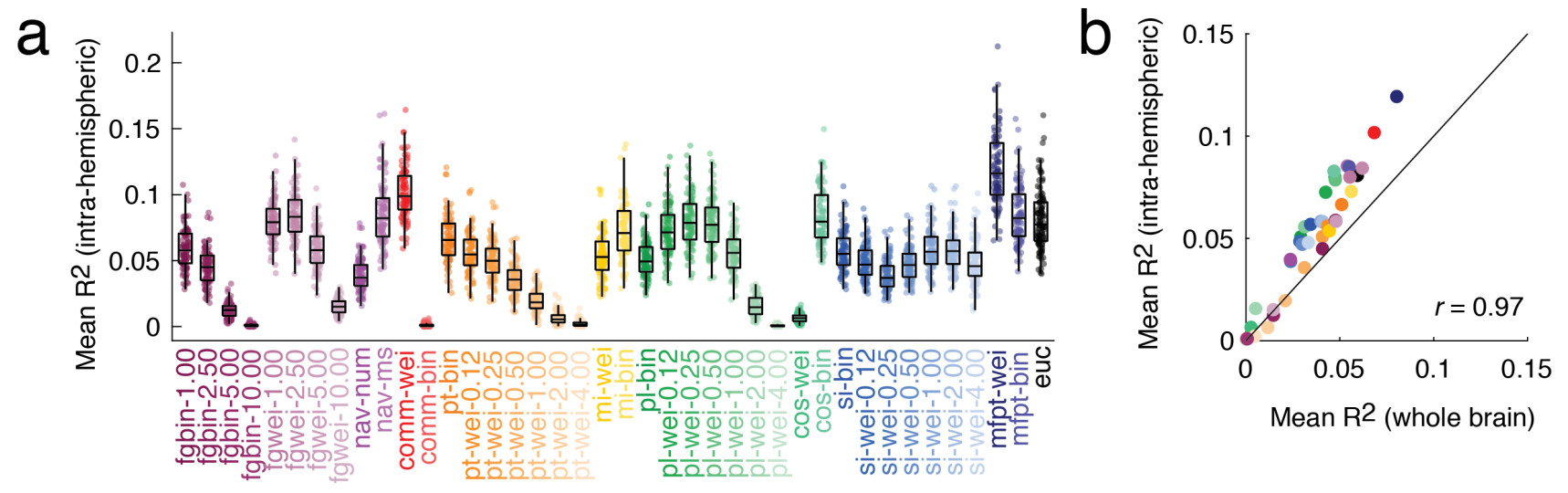

FIG. S2. Analysis of single hemisphere instead of whole-brain data. (a) Variance in single-hemisphere FC weights explained by factors. Each point represents a subject. To obtain these data, we generated predictors using SC data from the right and left hemispheres separately. Then we used these data to make predictions about FC data from the same hemisphere. Here, we plot the average variance explained over right and left hemispheres. (b) Mean $R^{2}$ estimated using whole-brain versus single-hemisphere data. The black line is an identity line. Note that single-hemisphere data results in improved fits. 


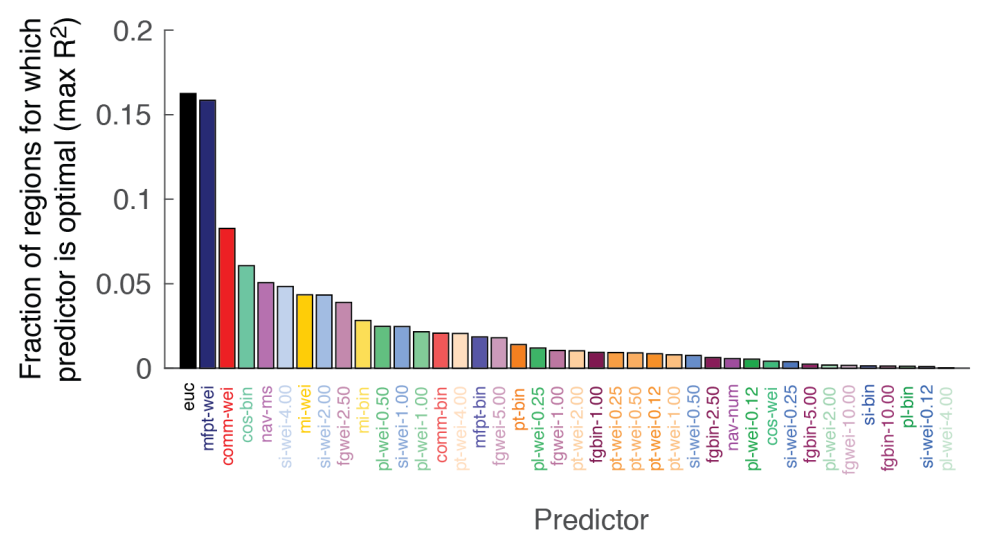

FIG. S3. Frequency of optimal predictors for modeling local structure-function relationships. Frequency across subjects and nodes with which each of the 40 predictors best-explained regional patterns of FC. 
a

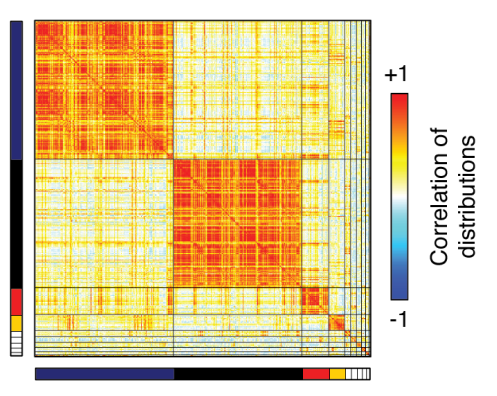

\section{C}
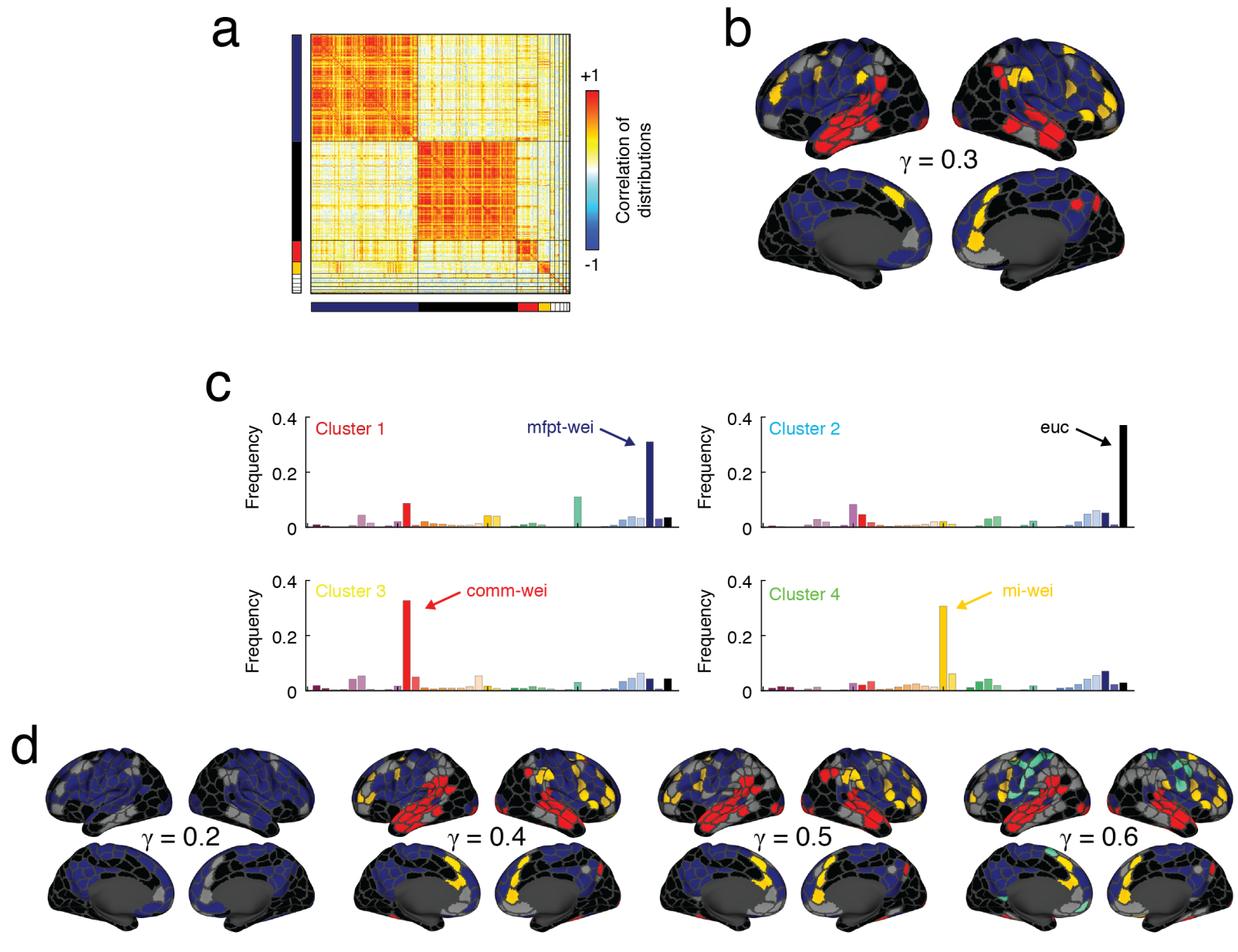

FIG. S4. Cluster analysis of correlated regional feature vectors. For each brain region we obtained a $40 \times 1$ feature vector whose elements denoted the frequency with which each of the predictors was "optimal" in the HCP dataset. We then computed the region-by-region correlation matrix and used modularity maximization to cluster this matrix and, again, to obtain consensus communities. (a) Correlation matrix ordered by consensus communities. Here we only label the four largest communities (red, cyan, yellow, green). (b) Consensus community assignments projected onto the cortical surface. (c) Mean feature vector for each of the four largest communities. $(d)$ For completeness, we show communities estimated at different resolutions ( $\gamma$ values). 
a

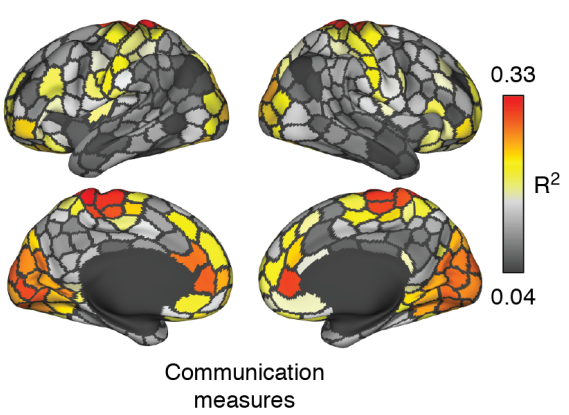

C

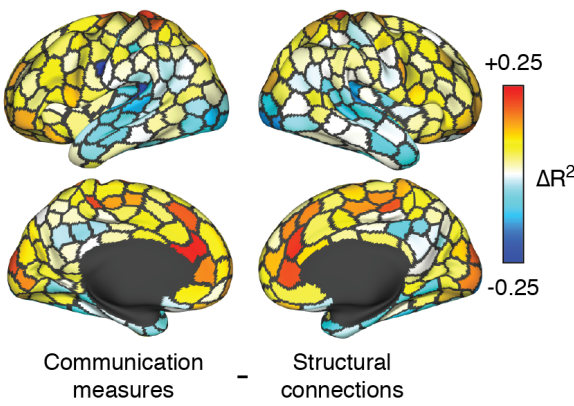

b

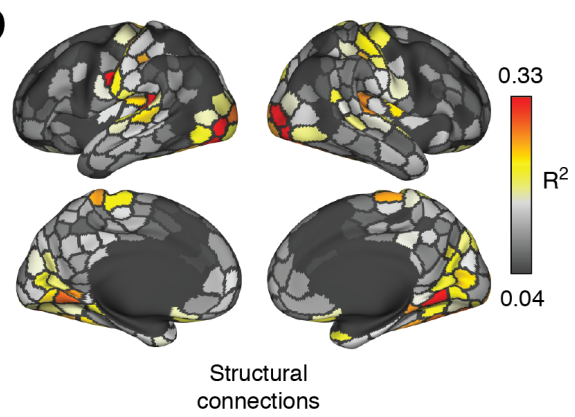

d

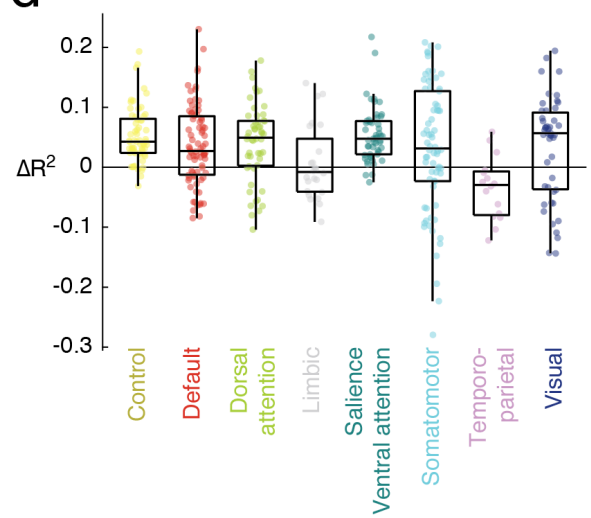

FIG. S5. Comparison of communication measures and structural connectivity in predicting FC. In the main text we derived a series of communication measures from sparse structural connectivity (SC) data to predict regional FC patterns. Here, we compare the results from the communication measures with the results obtained from using the structural connections directly. In this analysis, we create a "mask" for each region and subject of its structural connections to other regions. We then extract the weights of those structural and functional connections and fit a linear model to explain the functional connection weights in terms of the structural weights. This results in an $R^{2}$ value for each region and subject. (a) Variance explained using communication measures averaged across subjects. (b) Variance explained using structural connections alone averaged across subjects. $(c)$ Regional differences in variance explained. Warmer and cooler colors indicate regions whose FC is better predicted using the communication measures and structural connectivity, respectively. ( $d$ ) Data from panel $c$ but displayed by brain system.

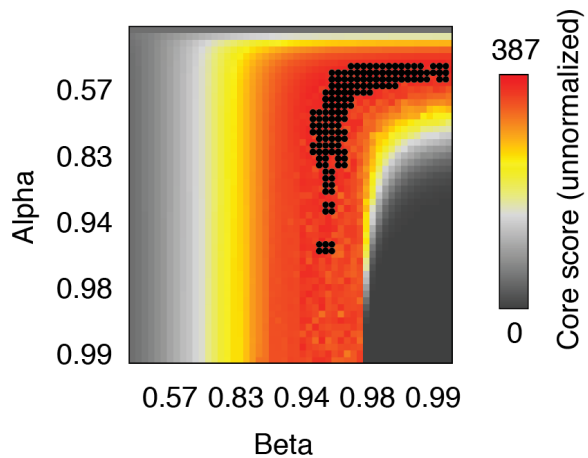

FIG. S6. Core-periphery analysis. Core score estimates at every point in parameter space. Black points indicate parameter values (top $5 \%$ of core scores) that were included in the core-periphery analysis. 


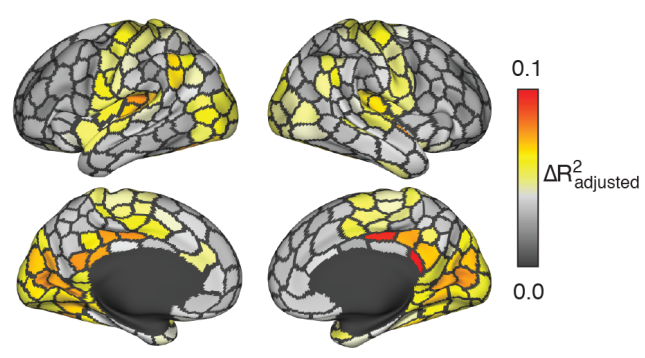

FIG. S7. Regional improvement in adjusted $R^{2}$. In the main text we reported the change in variance explained, $\Delta R^{2}$ as a result of including two predictors. In general, we might expect increases in $R^{2}$ simply due to the inclusion of a second parameter. Here, we show an analogous plot using the adjusted $R^{2}$ measure, which takes into account the number predictors. Specifically, we calculated $R_{\text {adjusted }}^{2}=1-\frac{\left(1-R^{2}\right)(N-1)}{N-p-1}$ for each region for both the one- and two-term models. Here, $N=399$ is the number of samples ( $N_{\text {regions }}-1$ because we exclude self-connections) and $p$ is the number of predictors in the model and is equal to $p=2$ and $p=3$ for the one- and two-predictor models (the additional parameter is from the intercept). In general, we find excellent correspondence between $\Delta R^{2}$ and $\Delta R_{\text {adjusted }}^{2}\left(r=0.99, p<10^{-15}\right)$. These observations suggest that the change in variance explained is not likely driven simply by an increase in the number of parameters.
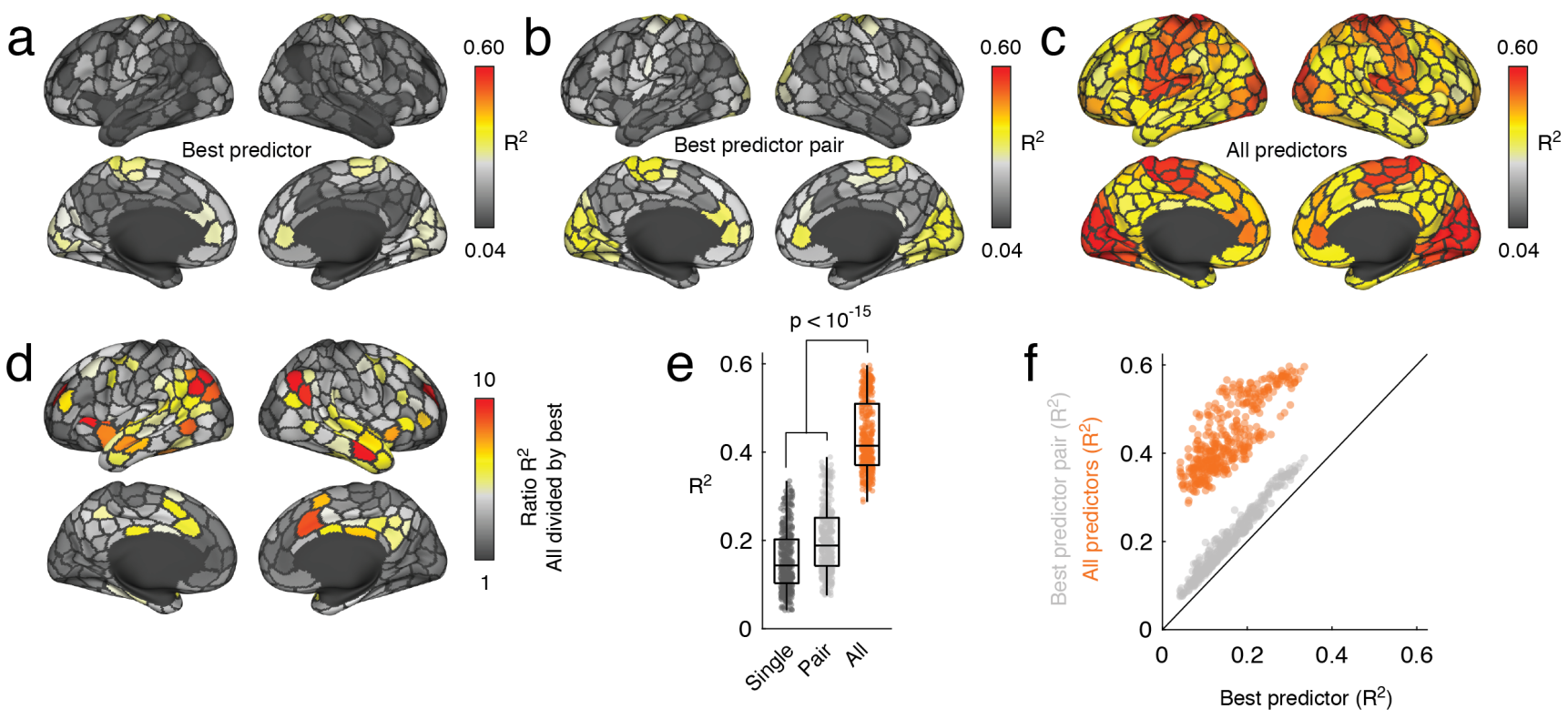

FIG. S8. Predicting FC using all measures. In the main text we predicted regional FC using metrics derived from SC matrices. Specifically, we focused on the variance explained by the best individual predictor $(a)$ and the best pair of predictors (b). Here, we repeat this analysis but using all predictors to predict regional FC. (c) The variance explained at each region after using all predictors. ( $d$ ) Ratio of variance explained using the best pair of predictors (panel $b$ ) and all predictors $(c)$. Note that the peak increases fall within default mode (interparietal sulcus; posterior cingulate; precuneus) and salience/ventral attention (anterior cingulate). (e) Boxplot of regional variance explained. $(f)$ Scatterplot of regional variance explained with the single best predictor versus the best pair of predictors (grey) and all predictors (orange). The black line is the identity line. 

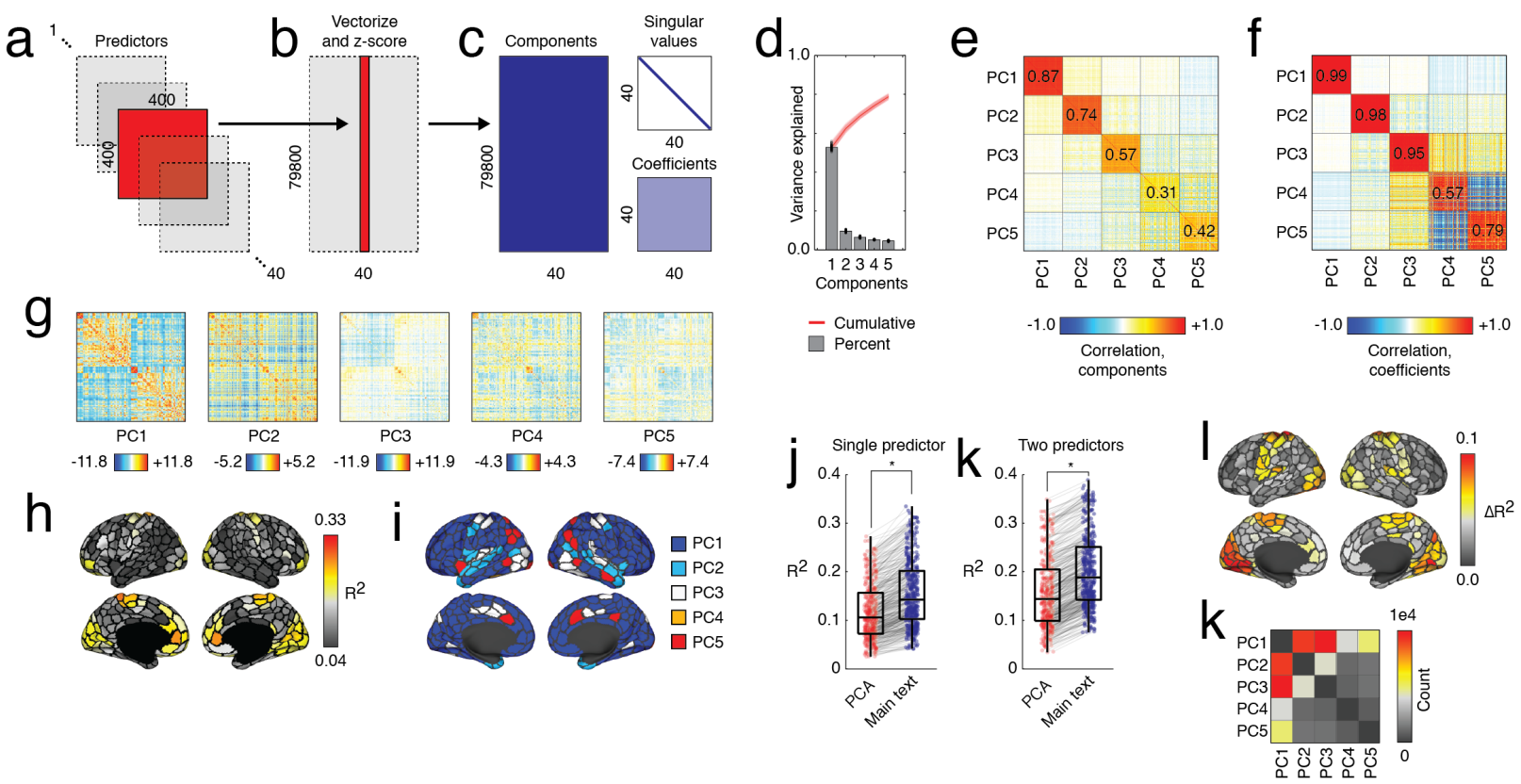

FIG. S9. Predicting FC using all PCs. In the main text we predicted regional patterns of FC using a series of predictors and later identified pairs of predictors that maximally improved these predictions. However, because predictors are correlated with one another, interpreting the results of this analysis may be difficult. Here, we use principal component analysis (PCA) to generate an orthonormal basis set from the predictors and use the resulting principal components (PCs) to predict FC. We performed PCA at the subject level using the 40 predictor matrices as input $(a)$. Each matrix was $(b)$ vectorized and z-scored before being decomposed into $(c)$ principal components, coefficients, and singular values. $(d)$ Here, we focus on the top five $\mathrm{PCs}$, which accounted for $>75 \%$ of variance across all subjects. We found that the top five PCs explain $52.7 \pm 1.3,9.7 \pm 0.5$, $6.5 \pm 0.4,5.3 \pm 0.2$ and $4.6 \pm 0.2$ percent variance, respectively. Despite PCA being performed on individual subjects, the PCs and coefficients were highly correlated across individuals. (e) We found that the similarity of the top five PCs across subjects was $0.87,0.74,0.57,0.31$, and 0.42 , respectively. This is despite the fact that the dimensionality of each $\mathrm{PC}$ was $[79800 \times 1]$. $(f)$ To similarity of coefficients from the top five PCs was $0.99,0.98,0.95,0.57$, and 0.79 , respectively. $(g)$ Group-averaged matrix representations of the first five PCs. We used PCs to make predictions about FC, fitting one linear model per region, per PC, and per subject. We identified for each subject the PC that yielded the greatest $R^{2}$ and averaged $R^{2}$ values across subjects. In panels, $h$ and $i$ we show $R^{2}$ values and the optimal PC projected onto cortical surface. We compared $R^{2}$ values reported in the main text with those generated using the PCA-based approach. We find that results from the main text outperform those generated using PCs (paired-sample t-test, $p<10^{-15}$ ). We present this comparison in panel $j$. As in the main text, we also tested the effect of combining pairs of predictors in the same multilinear model. Again, we found that the two-predictor models reported in the main text outperform the PCA approach (paired sample t-test, $p<10^{-15}$ ). A boxplot highlighting this comparison is shown in panel $k$. $(l)$ Increases in $R^{2}$ as a results of including an additional PC as a predictor. Interestingly, the combinations of predictors that lead to the greatest improvement tended to involve PC1 pairing with other components. $(k)$ A matrix plot of how frequently pairs of predictors were identified as the optimal model. 


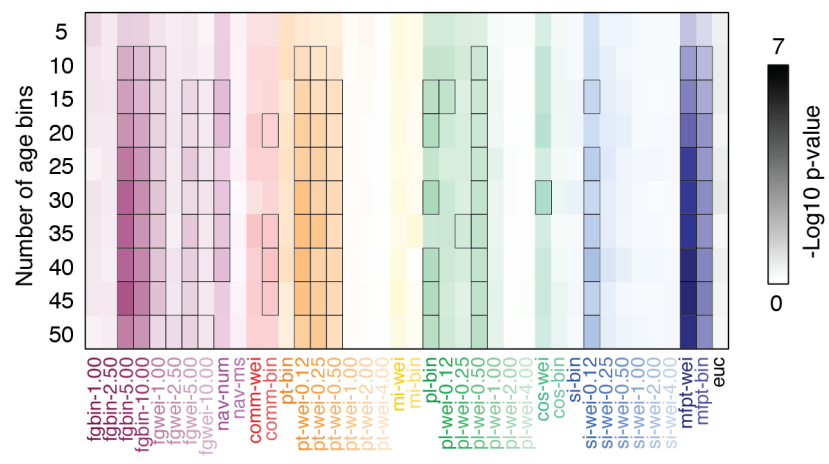

$=$ statistically significant, false discovery rate fixed at $1 \%(0.01)$

FIG. S10. Effect of bin size on the correlation of predictor frequency with age. In the main text we reported a correlation between the frequency with which given predictors are optimal for a given region and age. In that analysis, we partitioned subjects into ten age bins. Here, we show that these correlations persist across a broad range of age bins (from 5 to 50 in increments of 5$)$.

a

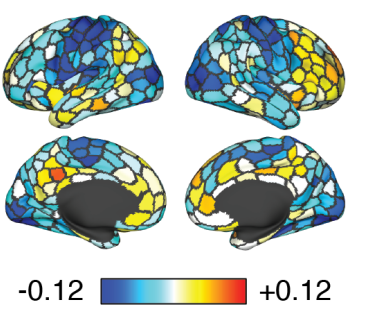

Correlation $\mathrm{R}^{2}$ with intelligence b

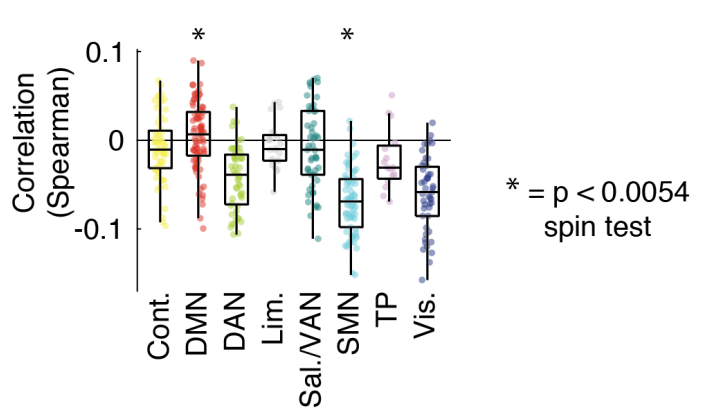

FIG. S11. Structure-function relationships are linked to intelligence. We calculated the regional correlation of the structure-function coupling $\left(R^{2}\right)$ with four measures of intelligence: (1) Wechsler Individual Achievement Test Composite score, (2-4) Wechsler Abbreviated Scale of Intelligence full scale IQ, verbal comprehension, and perceptual reasoning index. These scores were highly correlated $(r=0.72 \pm 0.13)$ and we report their mean correlation with $R^{2}$. (a) Spatial distribution of intelligence- $R^{2}$ associations projected onto cerebral cortex. (b) Correlation coefficients grouped by brain system. We compared the mean correlation of systems with a null distribution generated using a spin test (1000 permutations) and found that positive correlations were overly expressed in the default mode while negative correlations were overly expressed in the somatomotor network. 

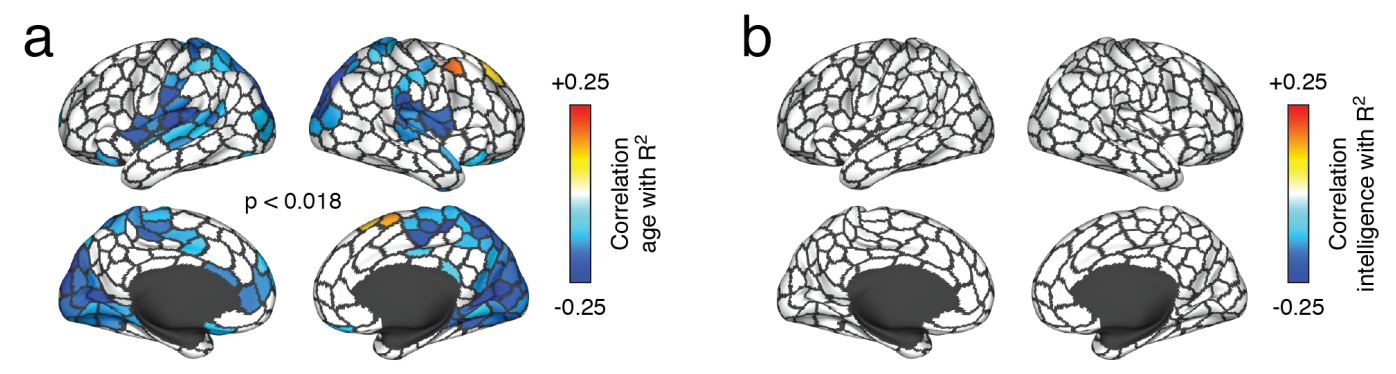

FIG. S12. Statistical thresholding of regional correlation maps. In the main text we computed the correlation of age and intelligence with the regional measures of structure-function coupling $\left(R^{2}\right)$. In those analyses, we performed statistical testing at the level of brain systems. That is, we identified systems whose average correlation was stronger - more positive or negative - than expected by chance. Here, we perform statistical testing at a regional level, correcting for multiple comparisons by adjusting the critical value to accommodate a false discovery rate of $q=0.05$, i.e. $5 \%$. In panel $a$, we show results of the age and $R^{2}$ correlation $\left(p_{a d j}=0.018\right)$. We note that no regions survive corrections for multiple comparisons in the case of correlation with intelligence.
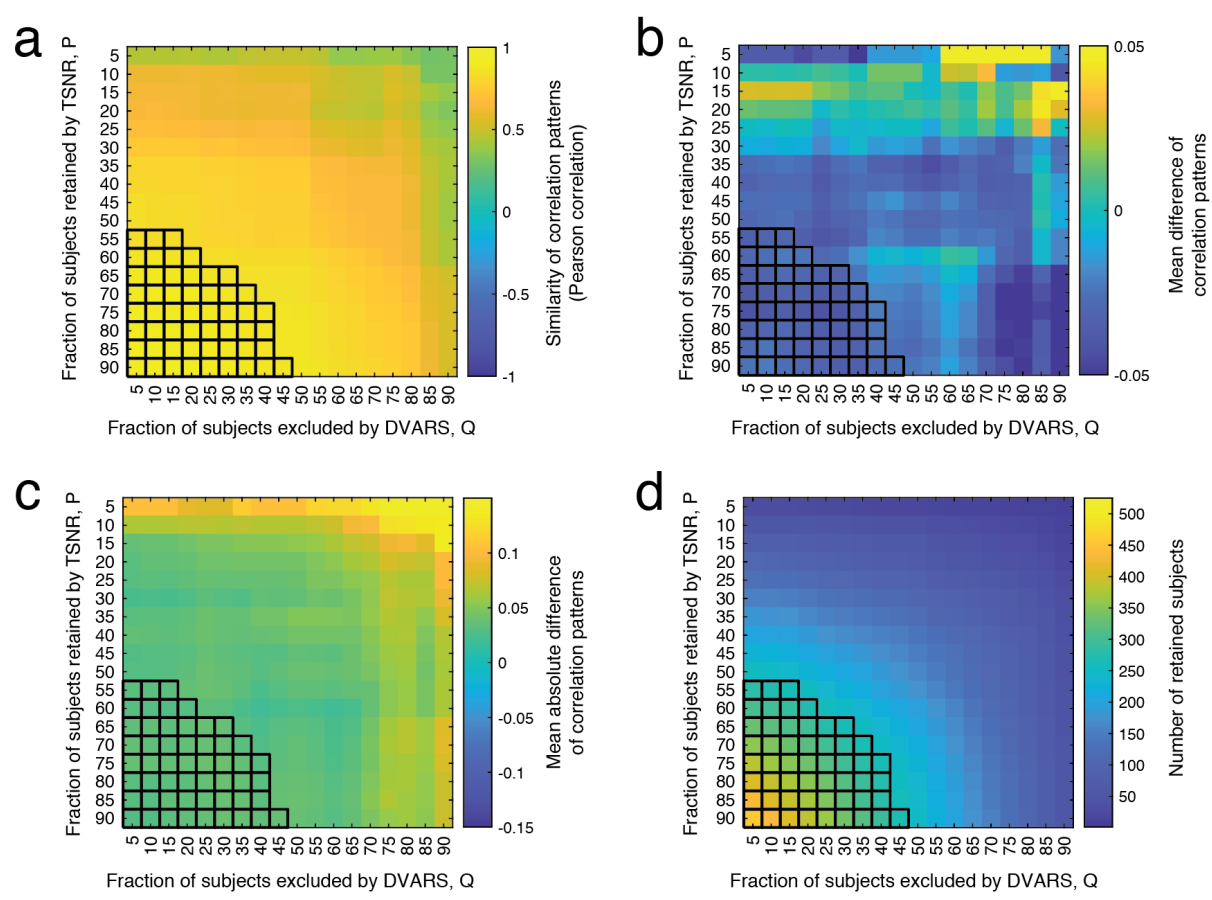

parameter combination that results in at least $0.5 \times$ Number of original participants

FIG. S13. Effect on correlation of regional $R^{2}$ versus age of motion-correction applied to NKI dataset. In the main text we reported a correlation between regional $R^{2}$ and age using the NKI dataset. Here we assess the effect of motion-correction on these results. Broadly, this strategy involved excluding data from subjects in the bottom $P \%$ based on their temporal signal to noise ratio (TSNR; mean BOLD signal divided by temporal standard deviation [126]) or was in the top $Q \%$ by DVARS (derivative of RMS over voxels; 124]). Of the remaining individuals, we regressed out both variables from their regional $R^{2}$ values and computed their correlation with age. Depending on the values of $P$ and $Q$ different subsets of subjects were retained. Here, we systematically varied these parameters are report: $(a)$ The similarity of the $R^{2}$ versus age correlation pattern with the pattern reported in the main text. $(b)$ The mean difference in regional correlation coefficients $\left(\frac{1}{400} \sum_{i=1}^{400}\left(r_{\text {motioncorrected }}-\right.\right.$ $\left.\left.r_{\text {original }}\right)\right)$. (c) The mean difference in absolute regional correlation coefficients $\left(\frac{1}{400} \sum_{i=1}^{400}\left(\left|r_{\text {motioncorrected }}\right|-\mid r_{\text {original }}\right) \mid\right)$. $(d)$ The total number of subjects retained. In each panel, we also plot parameter combinations that result in at least 263 subjects being retained (half of the original sample). 

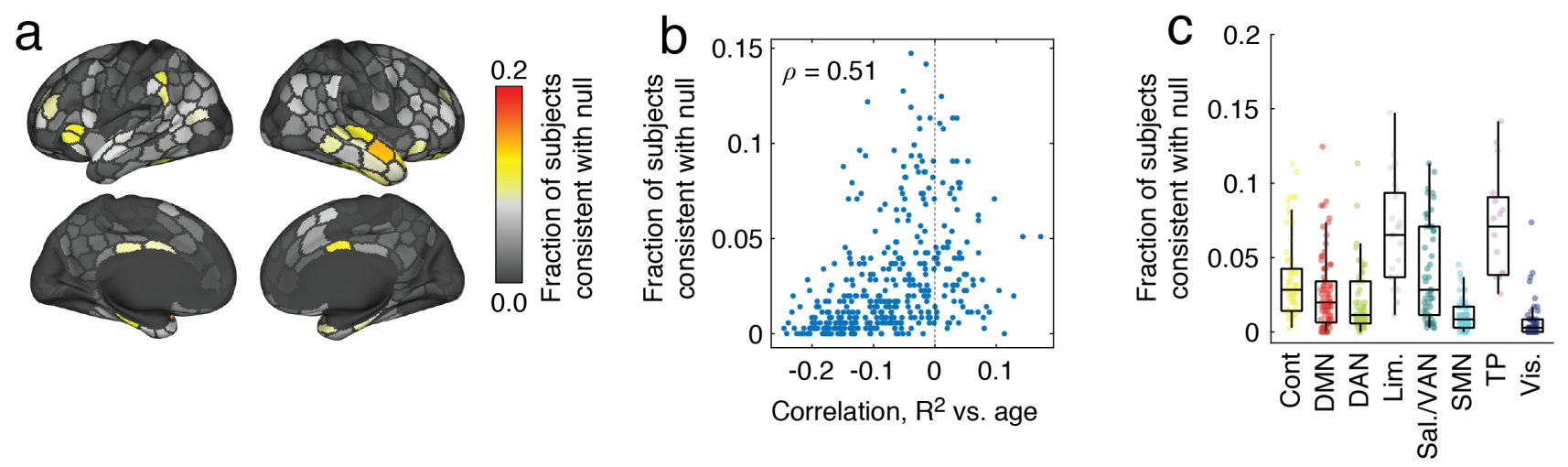

FIG. S14. Floor effect in NKI dataset. In the main text we reported a correlation between regional $R^{2}$ and age using the NKI dataset. Here we assess the possibility that the spatial pattern of these correlations may be driven, in part, by a floor effect. To test for this effect, we use a permutation-based null model. Regions whose $R^{2}$ values cannot be statistically distinguished from the null distribution are especially susceptible to a floor effect. In more detail, this procedure entailed the following. For each subject, we predicted their regional FC patterns using predictors derived from a randomized SC matrix (the order of its rows and columns were permuted). We then calculated and retained, for each region, its maximum $R^{2}$ across any of the predictors. We repeated this procedure 100 times for each subject, generating subject-specific null distributions. We compared the observed $R^{2}$ values with this null distribution (z-score) and identified regions whose z-statistic could not be distinguished statistically from the null distribution $(p \geq 0.05)$. We counted how frequently across subjects each of the 400 regions was part of this group, considering them to have already reached their floor $R^{2}$. We find that, on average, most regions exhibit $R^{2}$ values that are statistically greater than their theoretical floor $(96.8 \pm 1.5 \%)$. The specificity of regions that are approaching their $R^{2}$ floor is also poor; the region with the greatest frequency is consistent with the null distribution in $14.7 \%$ of subjects. Interestingly, and as the reviewer anticipated, the whole-brain pattern is similar to the correlation pattern reported in the main text $\left(\rho=0.51 ; p<10^{-15}\right)$, opening up the possibility that some of the age-related differences may be attributable to a floor effect. (a) Fraction of subjects in which a region's $R^{2}$ is not distinguishable from the null distribution. We show these values projected onto the cortical surface. (b) Values from panel $a$ with the regional $R^{2}$ versus age correlation coefficients. ( $c$ ) Values from panel $a$ grouped by system. 